\title{
Theory of quantum impurities in spin liquids
}

\section{Citation}

Kolezhuk, Alexei, Subir Sachdev, Rudro R. Biswas, and Peiqiu Chen. 2006. "Theory of Quantum Impurities in Spin Liquids." Physical Review B 74 (16). https://doi.org/10.1103/ physrevb.74.165114.

\section{Permanent link}

http://nrs.harvard.edu/urn-3:HUL.InstRepos:41417244

\section{Terms of Use}

This article was downloaded from Harvard University's DASH repository, and is made available under the terms and conditions applicable to Other Posted Material, as set forth at http:// nrs.harvard.edu/urn-3:HUL.InstRepos:dash.current.terms-of-use\#LAA

\section{Share Your Story}

The Harvard community has made this article openly available.

Please share how this access benefits you. Submit a story.

\section{Accessibility}




\title{
Theory of quantum impurities in spin liquids
}

\author{
Alexei Kolezhuk, Subir Sachdev, Rudro R. Biswas, and Peiqiu Chen \\ Department of Physics, Harvard University, Cambridge MA 02138
}

(Dated: June 9, 2018)

\begin{abstract}
We describe spin correlations in the vicinity of a generalized impurity in a wide class of fractionalized spin liquid states. We argue that the primary characterization of the impurity is its electric charge under the gauge field describing singlet excitations in the spin liquid. We focus on two gapless $\mathrm{U}(1)$ spin liquids described by $2+1$ dimensional conformal field theories (CFT): the staggered flux (sF) spin liquid, and the deconfined critical point between the Néel and valence-bond-solid (VBS) states. In these cases, the electric charge is argued to be an exactly marginal perturbation of the CFT. Consequently, the impurity susceptibility has a $1 / T$ temperature dependence, with an anomalous Curie constant which is a universal number associated with the CFT. One unexpected feature of the CFT of the sF state is that an applied magnetic field does not induce any staggered spin polarization in the vicinity of the impurity (while such a staggered magnetization is present for the Néel-VBS case). These results differ significantly from earlier theories of vacancies in the sF state, and we explicitly demonstrate how our gauge theory corrects these works. We discuss implications of our results for the cuprate superconductors, organic Mott insulators, and graphene.
\end{abstract}

PACS numbers: $75.30 . \mathrm{Hx}, 75.40 . \mathrm{Cx}, 76.60 . \mathrm{Cq}, 74.25 . \mathrm{Ha}$

\section{INTRODUCTION}

The response of a strongly interacting electronic system to impurities has long been a fruitful way of experimentally and theoretically elucidating the subtle correlations in its many-body ground state wavefunction. The most prominent example is the Kondo effect, which describes the interplay between a variety of impurities with a spin and/or 'flavor' degree of freedom and a system of free fermions with either a finite ${ }^{1.2 .3}$ or vanishing ${ }^{4}$ density of states at the Fermi energy.

More recently, the impurity responses of a variety of 'non-Fermi-liquid' bulk states have been

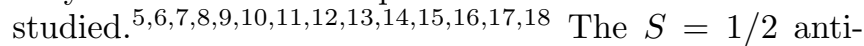
ferromagnetic spin chain generically has a critical ground state, and displays interesting universal characteristics in its response to impurities or boundaries ${ }^{6}$. Universality was also found in the general theory ${ }^{7.9 .16}$ of impurities in 'dimerized' quantum antiferromagnets in spatial dimensions $d \geq 2$ near a quantum critical point between a Néel state and a confining spin gap state. Such 'dimerized' antiferromagnets have an even number of $S=1 / 2$ spins per unit cell, and consequently their bulk quantum criticality is described within the conventional LandauGinzburg-Wilson (LGW) framework of a fluctuating Néel

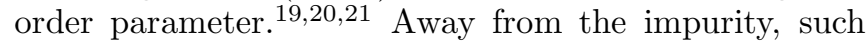
systems only have excitations which carry integer spin.

It is the purpose of this paper to extend the above theory ${ }^{7.9 .16}$ to fractionalized 'spin liquid' states in spatial dimensions $d \geq 2$ with neutral $S=1 / 2$ excitations ('spinons') in the bulk. Such spinon excitations carry gauge charges associated with an 'emergent' gauge force (distinct from the electromagnetic forces), typically with the gauge group $22.23 \quad Z_{2}$ or $\mathrm{U}(1)$, and we will argue shortly that such gauge forces play a key role in the response of spin liquid states to impurities. Earlier analyses $24,25,26,27,28$ of the influence of impurities in the
$\mathrm{U}(1)$ 'staggered-flux' spin liquid ignored the crucial gauge forces; we will comment in detail on the relationship of our results to these works in Section IIIB An analysis of impurities in a $Z_{2}$ spin liquid state was presented recently by Florens et al. 18 but for a particular situation in which a spin moment was strongly localized on an impurity and gauge forces could be safely neglected; we will comment further on their work in Section IVB

There are a number of experimental motivations for our work. A large number of experiments have studied $\mathrm{Zn}$ and $\mathrm{Ni}$ impurities in the cuprates,$\stackrel{29.30 .31 .32}{ }$ and much useful information has been obtained on the spatial and temperature dependence of the induced moments around the impurity. It would clearly be useful to compare these results with the corresponding predictions for different spin liquid states, and for states proximate to quantum critical points. We will show here that there are significant differences in the experimental signatures of the different candidates, and this should eventually allow clear discrimination by a comparison to experimental results. A second motivation comes from a recent nuclear magnetic resonance (NMR) study ${ }^{33}$ of the $S=1 / 2$ triangular lattice organic Mott insulator $\kappa-(\mathrm{ET})_{2} \mathrm{Cu}_{2}(\mathrm{CN})_{3}$, which possibly has a non-magnetic, spin singlet ground state. The NMR signal shows significant inhomogeneous broadening, indicative of local fields nucleated around impurities. Our theoretical predictions here for Knight shift around impurities should also assist here in selecting among the candidate ground states.

An important observations is that in situations with deconfinement in the bulk, the bulk spinons are readily available to screen any moments associated with an impurity atom (as has also been noted by Florens et al $l^{18}$ ). Moreover, for a non-magnetic impurity (such as Zn on a $\mathrm{Cu}$ site), there is no a priori reason for the impurity to acquire a strongly localized moment. Consequently, it is very useful to consider the case where the impurity has 
local net spin $S=0$. Naively, such a situation might seem quite uninteresting, as there is then no local spin degree of freedom which can interact non-trivially with the excitations of the spin liquid. Indeed, in a Fermi liquid, a non-magnetic impurity has little effect, apart from a local renormalization of Fermi liquid parameters, and there is no Kondo physics. However, in spin liquids the impurity can carry an electric gauge charge $Q$, and the primary purpose of this paper will be to demonstrate that a $Q \neq 0, S=0$ impurity displays rich and universal physics.

We will primarily consider $\mathrm{U}(1)$ spin liquids here: then an important dynamical degree of freedom is a $\mathrm{U}(1)$ gauge field $A_{\mu}$, where $\mu$ extends over the $d+1$ spacetime directions, including the imaginary time direction, $\tau$. Our considerations here can also be easily extended to $Z_{2}$ spin liquids, and this will be described later in Section IVB We normalize the $A_{\mu}$ gauge field such that the spinons have electric charges \pm 1 . In the popular $\mathrm{U}(1)$ gauge theories of antiferromagnets on the square lattice (which we will describe more specifically below), a vacancy will carry a gauge charge $Q= \pm 1$. Thus, a Zn impurity on the $\mathrm{Cu}$ square lattice site has $Q= \pm 1$. This can be understood by thinking of the impurity as a localized 'holon' in the doped antiferromagnet, which also carries such gauge charges 26 .

We will consider theories here with actions of the structure

$$
\mathcal{S}=\mathcal{S}_{b}+\mathcal{S}_{\text {imp }},
$$

where $\mathcal{S}_{b}$ is the bulk action of the spin liquid in the absence of any impurity, and $\mathcal{S}_{\text {imp }}$ represents the perturbation due to an impurity which we assume is localized near the origin of spatial coordinates, $x=0$. We will argue that the dominant term in $\mathcal{S}_{\mathrm{imp}}$ is the coupling of the impurity to the $\mathrm{U}(1)$ gauge field:

$$
\mathcal{S}_{\mathrm{imp}}=i Q \int d \tau A_{\tau}(x=0, \tau) .
$$

We will demonstrate that additional terms in the impurity action are unimportant or 'irrelevant'. The $\mathcal{S}_{\text {imp }}$ above can be regarded as the remnant of the spin Berry phase that characterized the impurity in the previous theory ${ }^{7.9 .16}$ of dimerized antiferromagnets; the latter Berry phase for a spin $S$ impurity was $i S$ times the area enclosed by the path mapped on the unit sphere by the time history of the impurity spin. An explicit reduction in the spinon formulation of the spin Berry phase to Eq. (1.2) was presented in Ref. 34 .

We will consider a variety of realizations of the bulk spin liquid $\mathcal{S}_{b}$ in this paper. Our primary results will be for $\mathrm{U}(1)$ algebraic spin liquids, 35 which are described by $2+1$ dimensional conformal field theories (CFT) and we specialize our presentation to these CFT cases in the remainder of this section. An algebraic spin liquid has gapless spinon excitations which interact strongly with the $A_{\mu}$ gauge field. An explicit realization appears in the deconfined quantum critical point $\frac{19,20.21}{}$ between Néel and valence bond solid (VBS) states, in which the spinons are relativistic bosons described by the $\mathrm{CP}^{N-1}$ field theory. Another is found in the 'staggered flux' ( $\mathrm{sF}$ ) phase of $\mathrm{SU}(N)$ antiferromagnets, where the spinons are Dirac fermions ${ }^{35,36,37.38}$. In all these cases, the algebraic spin liquid is described by a $2+1$ dimensional conformal field theory, and our primary purpose here is to describe the boundary conformal field theory that appears in the presence of $\mathcal{S}_{\text {imp }}$.

Our central observation, forming the basis of our results, is that $\mathcal{S}_{\mathrm{imp}}$ in Eq. (1.2) is an exactly marginal perturbation to the bulk conformal field theory. This non-renormalization is a consequence of $\mathrm{U}(1)$ gauge invariance, which holds both in the bulk and on the impurity. We will verify this non-renormalization claim in a variety of perturbative analyses of the conformal field theory. The claim can also be viewed as a descendant of the non-renormalization of the spin Berry phase term, found in Ref. 16.

The exact marginality of $\mathcal{S}_{\text {imp }}$ has immediate consequences for the response of the system to a uniform applied magnetic field $H$. The impurity susceptibility, $\chi_{\mathrm{imp}}$, defined as the change in the total bulk susceptibility due to the presence of the impurity, obeys

$$
\chi_{\text {imp }}=\frac{\mathcal{C}}{T}
$$

at finite temperature $T$ above a conformal ground state; this can be extended by standard scaling forms (as in Ref. 9) to proximate gapped or ordered phases, as we will describe in the body of the paper. We set $\hbar=k_{B}=1$ and absorb a factor of the magneton, $g \mu_{B}$, in the definition of the Zeeman field. With this, $\mathcal{C}$ is a dimensionless universal number, dependent only upon the value of $Q$, and the universality class of the bulk conformal field theory.

It is remarkable that the response of the impurity has a Curie-like $T$ dependence, albeit with an anomalous Curie constant $\mathcal{C}$ (which is likely an irrational number). This anomalous Curie response appears even though there is no spin moment localized on the impurity. In contrast, the earlier results for the LGW quantum critical point presented in Ref. 9 had an unscreened moment present and so a Curie response did not appear as remarkable. Here, it is due to the deformation of a continuum of bulk excitations by the impurity, and the $1 / T$ power-law is a simple consequence of the fact that $H$ and $T$ both scale as an energy. Indeed any other external field, coupling to a total conserved charge, will also have a corresponding universal $1 / T$ susceptibility.

A Curie-like response of an impurity in the staggered flux phase was also noted early on by Khalliulin and

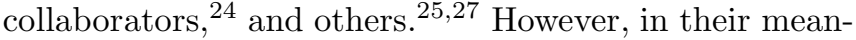
field analysis, they associated this response with a zero energy 'bound state', and hence argued that $\mathcal{C}=1 / 4$. As noted above, the actual interpretation is different: there is a critical continuum of excitations, and its collective boundary critical response has a Curie temperature dependence as a consequence of hyperscaling properties. 
Consequently, $\mathcal{C}$ does not equal the Curie constant of a single spin, and is a non-trivial number which is almost certainly irrational. We will discuss the earlier work more explicitly in Section IIIB

We will also consider the spatial dependence of the response to a uniform applied field, $H$, in the presence of an impurity, as that determines the Knight shift in NMR experiments. The uniform magnetization density induced by the applied field leads to a Knight shift $H K_{u}(x)$; at $T$ above a conformal ground state this obeys (the scaling form is also as in Ref. 9):

$$
K_{u}(x)=\frac{(T / c)^{d}}{T} \Phi_{u}(x T / c)
$$

where $c$ is the spinon velocity in the bulk (we assume the bulk theory has dynamic critical exponent $z=1$, and henceforth set $c=1), \Phi_{u}$ is a universal function, and the Knight shift is normalized so that

$$
\int d^{d} x K_{u}(x)=\chi_{\mathrm{imp}}
$$

The function $\Phi_{u}(y)$ has a power-law singularity as $y \rightarrow 0$, with the exponent determined by a 'boundary scaling dimension': this will be described in the body of the paper for the various cases.

In addition to the locally uniform Knight shift, an impurity in the presence of a uniform applied field also induces a 'staggered' moment which typically oscillates at the wavevector associated with a proximate magnetically ordered state. This leads to a staggered Knight shift, $H K_{s}(x)$, which we will also consider here. Such a staggered Knight shift does appear for the deconfined critical theory describing the Néel-VBS transition, and it has a spatial distribution associated with that of the Néel state. However, the response for the $\mathrm{U}(1) \mathrm{sF}$ spin liquid is dramatically different. One of our primary results is that for the scaling limit theory of the $\mathrm{U}(1) \mathrm{sF}$ spin liquid, an applied magnetic field in the presence of an impurity induces none of the many competing orders 37 associated with the spin liquid. Thus there is no analog of the 'staggered' Knight shift. A subdominant induction of competing orders can arise upon including irrelevant operators associated with corrections to scaling; the primary response, however, is just the induction of a ferromagnetic moment, which has a slowly-varying, spacedependent envelope in the vicinity of the impurity specified by $K_{u}(x)$.

Our conclusions above for the impurity response of the $\mathrm{U}(1) \mathrm{sF}$ spin liquid differ from the earlier meanfield theories $24,25,26,27,28$ They found an induced moment which had a strong oscillation between the two sublattices of the square lattice. We demonstrate here that this oscillation disappears in the continuum field theory which accounts for the gauge fluctuations. We are not aware of any reason why fluctuation corrections to the mean-field predictions should be considered small.

The outline of the remainder of the paper is as follows. In Section III we will consider the $\mathrm{CP}^{N-1}$ model field theory which describes the vicinity of the Néel-VBS transition. The U(1) sF spin liquid will then be described in Section III. Other spin liquids, not described by CFTs or by exactly marginal impurity perturbations, will be briefly discussed in Section IV Finally, experimental implications and a summary appear in Section $\nabla$

\section{II. $\mathrm{CP}^{N-1}$ MODEL}

The $\mathrm{CP}^{N-1}$ model describes the deconfined quantum critical point between the Néel and VBS states on the square lattice $\frac{19,20,21}{}$ It is a field theory of complex scalars $z_{\alpha}, \alpha=1 \ldots N$, with global $\mathrm{SU}(N)$ symmetry and a coupling to the $\mathrm{U}(1)$ gauge field $A_{\mu}$. Near the transition at which global $\mathrm{SU}(N)$ symmetry is broken we can work with the effective action

$$
\begin{aligned}
& \mathcal{S}_{b}=\int d^{D} y\left\{\left|\left(\partial_{\mu}-i A_{\mu}\right) z_{\alpha}\right|^{2}+s\left|z_{\alpha}\right|^{2}+\frac{u_{0}}{2}\left(\left|z_{\alpha}\right|^{2}\right)^{2}\right. \\
& \left.+\frac{1}{2 e_{0}^{2}}\left(\epsilon_{\mu \nu \lambda} \partial_{\nu} A_{\lambda}\right)^{2}\right\}
\end{aligned}
$$

where $y=(\tau, \vec{x})$ is a spacetime coordinate, $\mu$ extends over $D$ spacetime indices, $D$ is the dimension of spacetime, related to the spatial dimensionality, $d$, by

$$
D=d+1
$$

We are interested in the phases of the field theory in Eq. (2.1) as a function of the tuning parameter $s$. For $s \ll 0$ there is a magnetically ordered phase with global $\mathrm{SU}(N)$ symmetry broken, while for $s \gg 0$ we have a spin-gap phase with full $\mathrm{SU}(N)$ symmetry. We are especially interested in the conformally-invariant critical point which separates these phases. We will begin by reviewing the critical properties of the bulk action $\mathcal{S}_{b}$ alone in the following subsection, and describe impurity effects in the subsequent subsections.

\section{A. Bulk theory}

We will restrict our analysis here to the $\epsilon$ expansion where

$$
\epsilon=4-D
$$

This was carried out by Halperin et al $\underline{39}^{\underline{39}}$ some time ago, in a different physical context. This section will merely recast their results in our notation, using the fieldtheoretical formulation.

As noted by Halperin et al., a stable fixed point is obtained in the $\epsilon$ expansion only for sufficiently large values of $N$. However, it is expected that in the physical dimension of $\epsilon=1$, the fixed point may well be stable down to the needed values of $N$. Our purpose here is to understand the basic features of the second-order critical point, and its response to impurities: so we will assume 
that the value of $N$ is large enough to ensure stability of the fixed point. This will delineate the essential scaling structure of the impurity response, but is not expected to be quantitatively accurate.

It is also possible to analyze this model using the $1 / N$ expansion, directly in $D=3$. We choose not to present this here, because the results are very similar to the $\epsilon$ expansion, and the methods are closely related to those used for the $1 / N_{f}$ expansion in Section III.

The renormalization proceeds by defining renormalized fields and coupling constants $u$ and $f$ by

$$
\begin{aligned}
z_{\alpha} & =Z_{z}^{1 / 2} z_{R \alpha} \\
u_{0} & =\frac{\mu^{\epsilon} Z_{4}}{Z_{z}^{2} S_{D}} u \\
e_{0}^{2} & =\frac{\mu^{\epsilon} Z_{e}}{S_{D}} f
\end{aligned}
$$

where

$$
S_{D}=\frac{2}{\Gamma(D / 2)(4 \pi)^{D / 2}} .
$$

We work in the Lorentz gauge, in which the $A_{\mu}$ propagator is $\left(\delta_{\mu \nu}-p_{\mu} p_{\nu} / p^{2}\right) / p^{2}$. In this gauge the renormalization constants are

$$
\begin{aligned}
& Z_{4}=1+\frac{(N+4) u}{\epsilon}+\frac{6 f^{2}}{\epsilon u} \\
& Z_{e}=1+\frac{N f}{3 \epsilon} \\
& Z_{z}=1+\frac{3 f}{\epsilon}
\end{aligned}
$$

From this we can determine the anomalous dimension, $\eta_{z}$ of the $z_{\alpha}$; note that this is gauge dependent

$$
\begin{aligned}
\eta_{z} & =\mu \frac{d}{d \mu} \ln Z_{z} \\
& =-3 f
\end{aligned}
$$

where this is to be evaluated at the fixed point of the $\beta$ functions in Eq. (2.8) below.

The $\beta$ functions are

$$
\begin{aligned}
& \beta(u)=\mu \frac{d u}{d \mu}=-\epsilon u+(N+4) u^{2}+6 f^{2}-6 f u \\
& \beta(f)=\mu \frac{d f}{d \mu}=-\epsilon f+\frac{N}{3} f^{2}
\end{aligned}
$$

The renormalization of $\left|z_{\alpha}\right|^{2}$ determines the critical exponent $\nu$. This is associated with the renormalization constant

$$
Z_{2}=1+\frac{(N+4) u}{\epsilon}
$$

In this gauge, there is no contribution to $Z_{2}$ of order $f$. The critical exponent $\nu$ is given by

$$
\begin{aligned}
\frac{1}{\nu} & =2+\mu \frac{d}{d \mu} \ln \frac{Z_{2}}{Z_{z}} \\
& =2-(N+4) u+3 f,
\end{aligned}
$$

where, again, this is to be evaluated at the fixed point of the $\beta$ functions in Eq. (2.8).

Finally, we consider the scaling dimensions of gaugeinvariant operators which characterize the observable spin correlations. The Néel order parameter is defined by

$$
\phi_{a}=z_{\alpha}^{*} T_{\alpha \beta}^{a} z_{\beta}
$$

where $T^{a}$ is a $N \times N$ matrix which is a generator of $\mathrm{SU}(N)$. We define its anomalous dimension $\eta$ by

$$
\operatorname{dim}\left[\phi_{a}\right]=(D-2+\eta) / 2
$$

In the Lorentz gauge, $\phi_{a}$ has no additional renormalization from $A_{\mu}$ fluctuations at leading order in $\epsilon$. The anomalous exponent $\eta$ is then given, to this order, by

$$
\eta=D-2+2 \eta_{z}+2 u+\mathcal{O}\left(\epsilon^{2}\right)
$$

Note that the value of $\eta$ is gauge-invariant, while that of $\eta_{z}$ is not; the relationship (2.13) holds only in the Lorentz gauge.

We will also be interested in correlations of the magnetization density $M_{a}$. This is defined by the response of the system to a uniform and staggered magnetic fields $H_{u}$ and $H_{s}$, under which the action is modified by

$$
\begin{aligned}
\mid\left(\partial_{\tau}\right. & \left.-i A_{\tau}\right)\left.z\right|^{2} \rightarrow\left\{\left(\partial_{\tau}+i A_{\tau}\right) z_{\alpha}^{*}+H_{u}^{a} T_{\alpha \beta}^{a *} z_{\beta}^{*}\right\} \\
& \times\left\{\left(\partial_{\tau}-i A_{\tau}\right) z_{\alpha}-H_{u}^{a} T_{\alpha \gamma}^{a} z_{\gamma}\right\}-H_{s}^{a} \phi_{a} .
\end{aligned}
$$

The magnetization density is given by

$$
M_{a}=T \frac{\delta \ln \mathcal{Z}}{\delta H_{u a}}
$$

where $\mathcal{Z}$ is the partition function. Because $H_{u}$ couples to a conserved 'charge', it scales as an energy, and therefore

$$
\operatorname{dim}\left[M_{a}\right]=d
$$

\section{B. Impurity exponents}

We now turn to an analysis of spin correlations in the vicinity of the impurity. The general method is very similar to that followed in Ref. 16.

We assume here that monopole tunneling events remain irrelevant at the impurity at the quantum critical point, as they do in the bulk ${ }^{19.20 .21}$ Such monopole tunneling events are defined only in $d=2$, and so their scaling dimensions are not easily estimated in the $\epsilon$ expansion. However, the monopoles are irrelevant at large $N$ in the bulk because their action is proportional to $N$, and the same reasoning applies also in the presence of the impurity.

As in Refs. 9. 16, the staggered and uniform magnetizations operators $\left(\phi_{a}\right.$ and $\left.M_{a}\right)$ acquire additional renormalizations as they approach the impurity at $x=0$. Let us denote the corrections by modifying (2.12) to

$$
\operatorname{dim}\left[\phi_{a}(x \rightarrow 0)\right]=\operatorname{dim}\left[\phi_{a}\right]+\Delta_{\mathrm{imp}}^{\phi}
$$


and similarly for $M_{a}$. We will compute these additional renormalizations to order $\epsilon^{2}$ below, and find that to this order $\Delta_{\text {imp }}^{\phi}=\Delta_{\text {imp }}^{M}$.

It is also useful to restate the above results in the notation of Refs. 9 16. These we find that the excitations near the impurity are characterized by a spin operator $S_{a}$, where $a$ is the index of $\mathrm{SU}(N)$ generators, and we denoted its scaling dimension by $\eta^{\prime} / 2$, or, equivalently,

$$
\left\langle S_{a}(\tau) S_{b}(0)\right\rangle \sim \frac{\delta_{a b}}{|\tau|^{\eta^{\prime}}}
$$

at the quantum critical point. Assuming that $S_{a}$ is obtained as $\phi_{a}$ approaches the impurity, we obtain from (2.12) and (2.17) that

$$
\eta^{\prime}=D-2+\eta+2 \Delta_{\text {imp }}^{\phi}
$$

These scaling relations imply ${ }^{40}$ that at the quantum critical point the temperature dependence of the NMR relaxation rate is

$$
1 / T_{1} \sim T^{-1+\eta^{\prime}},
$$

near the impurity, while $1 / T_{1} \sim T^{d-2+\eta}$ in the bulk. Finally, we note that we can also express the above relations using the the operator product expansion

$$
\lim _{x \rightarrow 0} \phi_{a}(x, \tau) \sim \frac{S_{a}(\tau)}{|x|^{-\Delta_{\mathrm{imp}}^{\phi}}} .
$$

It is also useful to consider time-dependent correlations of the magnetization density, $M_{a}$, as one approaches the impurity. The initial guess would be that these acquire the additional anomalous dimension $\Delta_{\mathrm{imp}}^{M}$. However, we will see in our computations below that the dominant correlations are instead given by 'mixing' between the $\phi_{a}$ and $M_{a}$ operators near the impurity. As was also found in Ref. 9, the impurity Berry phase allows such mixing, and so the operator product expansion of $M_{a}$ near the impurity has a dominant term given by the same operator $S_{a}$ introduced above to describe the behavior of $\phi_{a}$. This assertion is encapsulated in the operator product expansion

$$
\lim _{x \rightarrow 0} M_{a}(x, \tau) \sim \frac{S_{a}(\tau)}{|x|^{d-\eta^{\prime} / 2}} .
$$

The relations (2.18), 2.21) and (2.22) are keys to our analysis, and will allow us to deduce the structure of spin correlations near the impurity. Their validity will be supported by a number of perturbative results we will obtain below.

Now we turn to a determination of the exponents $\Delta_{\mathrm{imp}}^{\phi}$ and $\Delta_{\text {imp }}^{M}$. These are most easily specified by determining the additional renormalization factor needed to cancel poles in $\epsilon$ in correlators of $\phi_{a}$ and $M_{a}$ as one approaches the impurity. The simplest correlator of $\phi_{a}$ which allows us to achieve this is the vertex operator

$$
\left\langle\phi_{a}(x=0, \omega=0) z_{\alpha}(k=0, \omega) z_{\beta}^{*}(k=0, \omega)\right\rangle,
$$
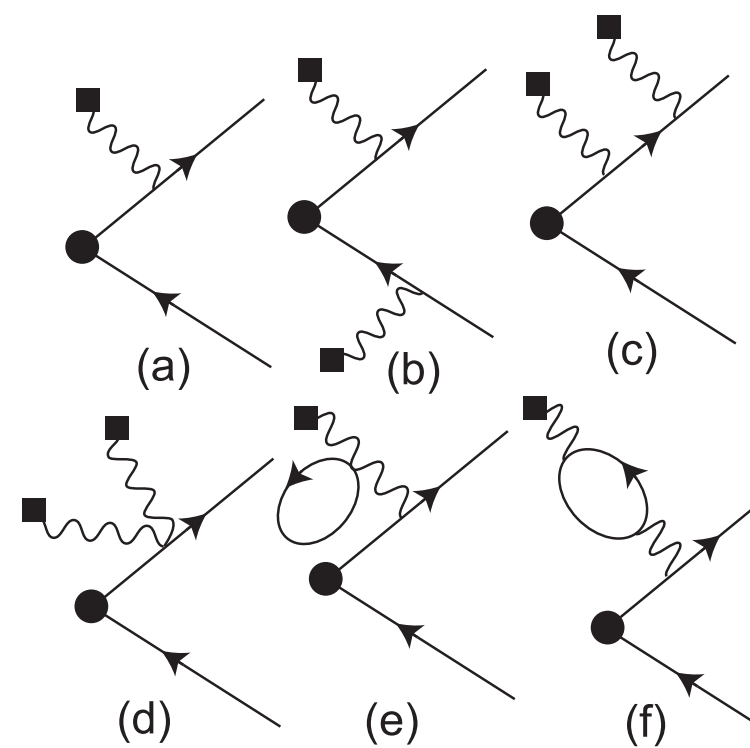

(b)

(c)

(d)

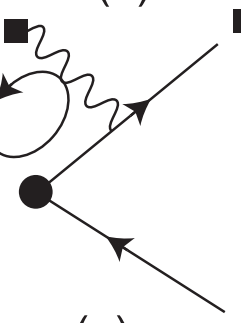

(e)
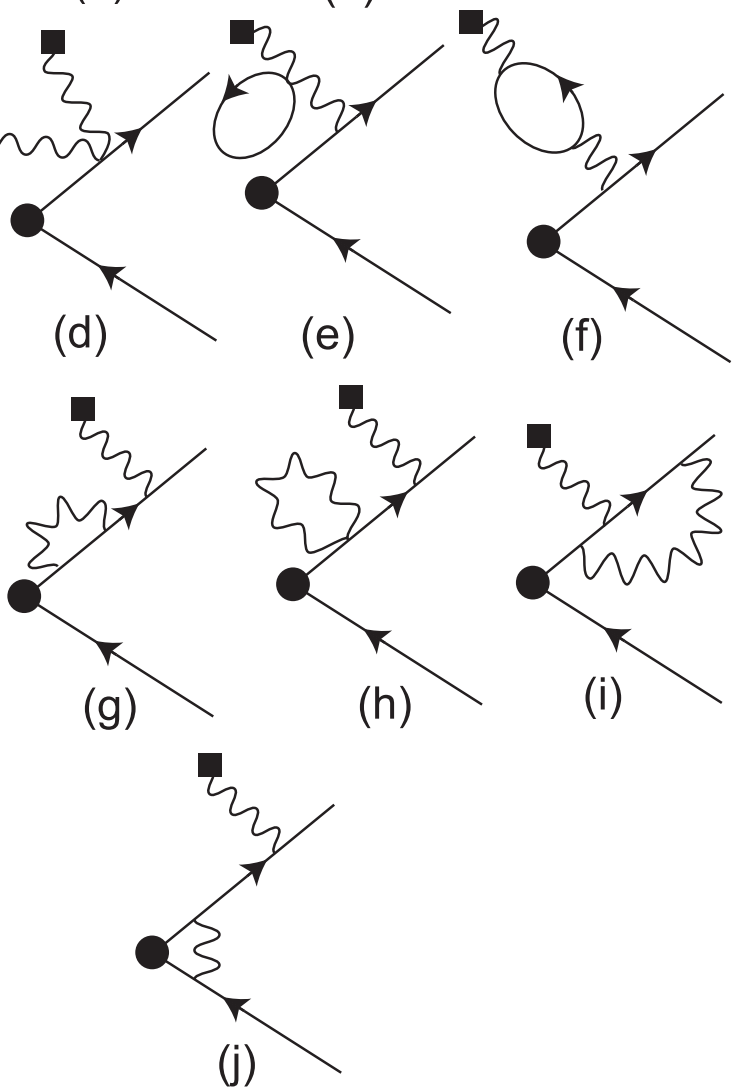

FIG. 1: Feynman diagrams which contribute to the impuritydependent renormalization of vertices such as those in Eq. (2.23). The full line is the $z_{\alpha}$ propagator, the wavy line is the $A_{\mu}$ propagator, and the filled square is the source term in $\mathcal{S}_{\text {imp }}$ (also in Fig. 21).

where the external $z_{\alpha}$ legs will be truncated, and similarly for the $M_{a}$. Here the external frequency $\omega$ is needed to control the infra-red singularities.

The leading contribution to (2.23), at order $\epsilon$ is shown in Fig. 11(a) and it renormalized the vertex by the factor

$$
2 i Q e_{0}^{2} \omega \int \frac{d^{d} q}{(2 \pi)^{d}} \frac{1}{q^{2}\left(q^{2}+\omega^{2}\right)}
$$

The same expression also applies to the renormalizations of the vertex associated with $M_{a}$. The $q$ integral is easily evaluated, and no pole in $\epsilon$ appears; this is fortunate, because the expression in (2.24) is purely imaginary.

The determination of the $\mathcal{O}\left(\epsilon^{2}\right)$ corrections involves a more difficult computation. There are a number of 
(a)

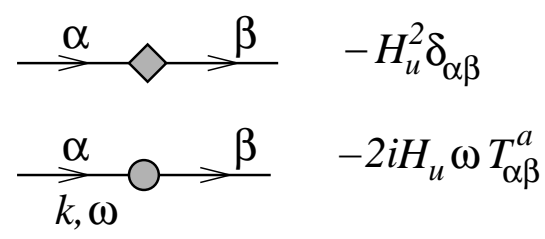

(c)

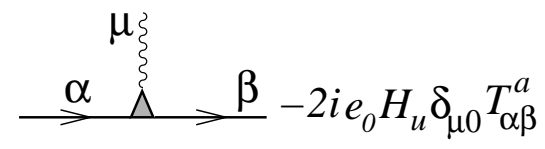

(d)

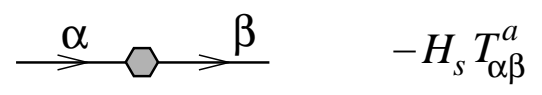

(e)

$$
\min _{k, \omega}^{\mu}-i e_{0} Q \delta_{\mu 0} \delta(\omega)
$$

FIG. 2: Vertices appearing in the calculation of response to applied field; $H_{u}$ and $H_{s}$ denote uniform and staggered magnetic fields, respectively.

graphs which contribute, shown in Fig. 1 Fig. 1(b)) is simply the square of Fig. I(a), and so contributes no pole in $\epsilon$. The value of Fig. 1(c) (and its symmetry related partner) is

$$
\begin{array}{r}
-8 Q^{2} e_{0}^{4} \omega^{2} \int \frac{d^{d} q}{(2 \pi)^{d}} \frac{d^{d} k}{(2 \pi)^{d}} \frac{1}{q^{2}\left(q^{2}+\omega^{2}\right)\left(k^{2}+\omega^{2}\right)(q+k)^{2}} \\
=\text { no pole in } \epsilon .
\end{array}
$$

The contribution of Fig. 1 (and its symmetry related partner), for both the $\phi_{a}$ and $M_{a}$ vertices is

$$
\begin{gathered}
2 Q^{2} e_{0}^{4} T_{\alpha \beta}^{a} \int \frac{d^{d} q}{(2 \pi)^{d}} \frac{d^{d} k}{(2 \pi)^{d}} \frac{1}{q^{2}\left(k^{2}+\omega^{2}\right)(q+k)^{2}} \\
=Q^{2} T_{\alpha \beta}^{a} \frac{4 \pi^{2} f^{2}}{\epsilon}+\ldots
\end{gathered}
$$

The diagrams Fig. 1(e-j) are all purely imaginary, so it must be that the poles in $\epsilon$ in their sum cancel. We have verified that this is indeed the case. Figs. I (e,f) yield a pole in $\epsilon$ which cancels against the second order contribution from Fig. I (b) after the charge renormalization in (2.6) is accounted for. The self-energy and vertex corrections in Figs. 1(g-i) cancel against each other, as usual. And finally, Fig प(j) represents an 'interference' between two first order contributions, the bulk renormalization of the vertex and the finite impurity contribution in Fig. I(a), and so requires no additional renormalizations. Collecting these results, and using the fixed point value of $f$ from (2.8), we obtain

$$
\Delta_{\text {imp }}^{\phi}=\Delta_{\text {imp }}^{M}=-\frac{72 \pi^{2} Q^{2}}{N^{2}} \epsilon^{2}+\mathcal{O}\left(\epsilon^{3}\right),
$$

although $\Delta_{\text {imp }}^{\phi}$ and $\Delta_{\text {imp }}^{M}$ are not expected to be equal at higher orders. (a)

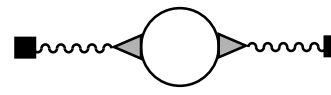

(b)

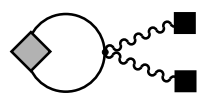

(c)

(d)

(e)

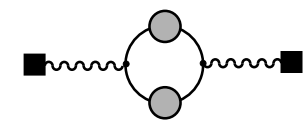

(f)

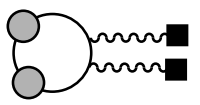

(g)

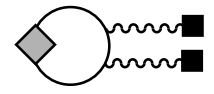

(h)

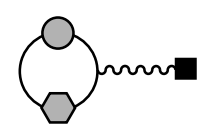

(i)
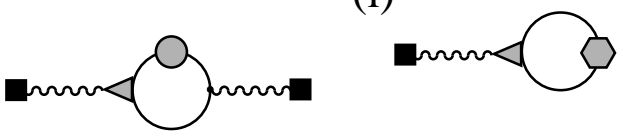

FIG. 3: Feynman diagrams determining the leading order contribution to the free energy in presence of uniform (diagrams (a)- (g)) and staggered (diagrams (h), (i)) magnetic fields.

\section{Linear response to a uniform applied field}

We will be interested in the spatially dependent magnetization response to an external static uniform magnetic field $H_{u}=H$. Let us start with the calculation of the impurity susceptibility $\chi_{\mathrm{imp}}$, which characterizes the total excess magnetization arising due to the impurity in response to the applied field.

\section{Impurity susceptibility}

The susceptibility can be defined as

$$
\chi_{\mathrm{imp}}=\frac{T}{N^{2}-1} \sum_{a} \frac{\delta^{2} \ln \mathcal{Z}}{\delta H_{u a} \delta H_{u a}},
$$

where the prefactor stems from averaging over the "spin" space of SU(N) generators. Using the action (2.1), modified according to (2.14), one can see that the presence of a uniform magnetic field leads to three new vertices shown in Fig. 2(a-c). The impurity-related diagrams must contain at least one impurity "source" vertex shown in Fig. $2(\mathrm{e})$.

It is easy to show that the leading order correction to the free energy is given by the diagrams of Fig. 3(a-g). Notice that the leading contribution is of the second order in the impurity charge $Q$, since all first-order diagrams happen to be odd in Matsubara frequency and thus are identically zero. The $A_{\mu}$ propagator enters those diagrams only as $D_{\mu 0}(k, \omega=0)=\delta_{\mu 0} / k^{2}$, and the latter expression is valid for any choice of gauge which ensures that our final results will be gauge-independent. Summing up the contributions of the diagrams (a-g) of Fig. 
3] one obtains

$$
\begin{aligned}
\chi_{\mathrm{imp}} & =2 Q^{2}\left(e_{0}^{2}\right)^{2} \widetilde{S} \int \frac{d^{d} k}{(2 \pi)^{d}} \frac{d^{d} k^{\prime}}{(2 \pi)^{d}} \frac{1}{\left(k^{\prime}\right)^{4}} g\left(\varepsilon_{k}, \varepsilon_{k+k^{\prime}}\right), \\
g(x, y) & =T \sum_{\omega_{n}}\left\{\frac{2}{\left(\omega_{n}^{2}+x^{2}\right)\left(\omega_{n}^{2}+y^{2}\right)}+\frac{1}{\left(\omega_{n}^{2}+x^{2}\right)^{2}}\right. \\
& -\frac{4 \omega_{n}^{2}}{\left(\omega_{n}^{2}+x^{2}\right)^{3}}+\frac{8 \omega_{n}^{4}}{\left(\omega_{n}^{2}+x^{2}\right)^{2}\left(\omega_{n}^{2}+y^{2}\right)^{2}} \\
& -\frac{16 \omega_{n}^{2}}{\left(\omega_{n}^{2}+x^{2}\right)^{2}\left(\omega_{n}^{2}+y^{2}\right)}+\frac{16 \omega_{n}^{4}}{\left(\omega_{n}^{2}+x^{2}\right)^{3}\left(\omega_{n}^{2}+y^{2}\right)} \\
& \left.-\frac{4 \omega_{n}^{2}}{\left(\omega_{n}^{2}+x^{2}\right)^{2}\left(\omega_{n}^{2}+y^{2}\right)}\right\},
\end{aligned}
$$

where $\varepsilon_{k} \equiv|k|$, and the sum in $g(x, y)$ runs over bosonic Matsubara frequencies $\omega_{n}=2 \pi n T$. The overall factor of $\widetilde{S}$ comes from a trace over the components of the field $z$ and is defined as

$$
\widetilde{S}=\frac{1}{N^{2}-1} \operatorname{tr} \sum_{a=1}^{N^{2}-1}\left(T^{a}\right)^{2} \equiv \frac{\widetilde{\mathcal{C}}}{N^{2}-1},
$$

where $\widetilde{\mathcal{C}}$ is the eigenvalue of the Casimir operator of $\mathrm{SU}(\mathrm{N})$ which depends on the specific representation of the group. In the $\mathrm{SU}(2)$ case, this factor takes the familiar form $\widetilde{S}=S(S+1) / 3$. It is worth noting that all the diagrams are symmetric with respect to the exchange of $k$ and $k+k^{\prime}$, so only the symmetric part of $g(x, y)$ yields a finite contribution to the susceptibility.

Obviously, the expression (2.29) contains a singularity at $k, k^{\prime} \rightarrow 0$. At $T=0$ this singularity is formally unimportant, since at $T \rightarrow 0$ the function $g(x, y)$ becomes fully antisymmetric:

$$
\lim _{T \rightarrow 0} g(x, y) \rightarrow \frac{x-y}{2 x y(x+y)^{2}},
$$

so at $T=0$ the impurity susceptibility is identically zero. Alternatively, one may notice that the expression under the sum sign in (2.29) is a full derivative in $\omega_{n}$, which implies that $\chi_{\mathrm{imp}}$ vanishes at $T=0$ when the sum in $\omega_{n}$ is replaced by an integral.

At finite $T$, however, the symmetric part $\widetilde{g}(x, y)=$ $1 / 2(g(x, y)+g(y, x))$ is finite:

$$
\widetilde{g}(x, y)=\frac{x\left(3 y^{2}+x^{2}\right) \cosh (y / 2 T)}{16 x y\left(x^{2}-y^{2}\right) T^{2} \sinh ^{2}(y / 2 T)}+(x \leftrightarrow y),
$$

and the infrared singularity becomes dangerous (the integrand in (2.29) behaves as $k^{-4}\left(k^{\prime}\right)^{-4}$ at $k, k^{\prime} \rightarrow 0$ ). Such finite- $T$ infrared divergencies in massless theories are well-known ${ }^{41}$ and can be cured by incorporating the thermal "screening mass" into free propagators. The thermal masses $m_{p}$ and $m_{b}$ (for the "photon" $A_{\mu}$ and for the boson $z$, respectively) can be easily found to the (a)

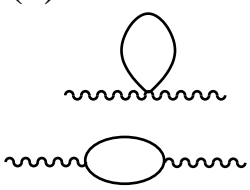

(b)

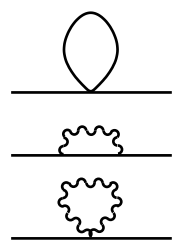

FIG. 4: Self-energy diagrams determining the thermal screening masses (2.33) for the "photon" $A_{\mu}$ (a) and for $z$-boson (b).

first order in the coupling constants $e_{0}^{2}$ and $u_{0}$ by computing the temperature-dependent contribution to the selfenergy diagrams shown in Fig. 4

$$
m_{p}^{2}=\frac{e_{0}^{2} N}{6} T^{2}, \quad m_{b}^{2}=\left\{\frac{(N+1) u_{0}}{12}+\frac{e_{0}^{2}}{4}\right\} T^{2} .
$$

At the critical point $e_{0}^{2}$ and $u_{0}$ are both proportional to $\epsilon=4-D$, so that we can write

$$
m_{p, b}^{2}=\lambda_{p, b} T^{2} \epsilon,
$$

where $\lambda_{p, b}$ can be found from the beta-functions (2.8): one obtains $\lambda_{p}=4 \pi^{2}$, and the expression for $\lambda_{b}$ is rather complicated $^{42}$ but simplifies in the large- $N$ limit:

$$
\lambda_{p}=4 \pi^{2}, \quad \lambda_{b} \rightarrow \pi^{2} / 6, \quad N \rightarrow \infty .
$$

Taking into account the thermal masses amounts to replacing $\left(k^{\prime}\right)^{2} \mapsto\left(k^{\prime}\right)^{2}+m_{p}^{2}$ and $x^{2} \mapsto x^{2}+m_{b}^{2}, y^{2} \mapsto$ $y^{2}+m_{b}^{2}$ in Eq. (2.29). It is easy to see that the main contribution to the integral in (2.29) comes from small momenta $k, Q \lesssim m_{b, p} \ll T$, so one can replace $\widetilde{g}(x, y)$ by

$$
\begin{aligned}
\lim _{y, x \rightarrow 0} \widetilde{g}(x, y) & \mapsto \frac{1 / 2}{\left(x^{2}+m_{b}^{2}\right)^{2}}+\frac{1 / 2}{\left(y^{2}+m_{b}^{2}\right)^{2}} \\
& +\frac{2}{\left(x^{2}+m_{b}^{2}\right)\left(y^{2}+m_{b}^{2}\right)} .
\end{aligned}
$$

This means that the main contribution to the susceptibility comes from the zero Matsubara frequency $\left(\omega_{n}=0\right)$ part of the expression (2.29) and thus is determined by the first two diagrams in Fig. 3 Evaluating the momentum integrals in (2.29) in $d=3$, and substituting $e_{0}^{2}$ by its fixed-point value $\frac{24 \epsilon \pi^{2}}{N}$, one readily obtains the Curielike law (1.3) for the impurity susceptibility, with the anomalous Curie constant $\mathcal{C}$ given by

$$
\mathcal{C}=\frac{9 \epsilon Q^{2} \widetilde{S} \lambda_{p}^{2}}{2 \pi^{2} N^{2}}\left\{\frac{1}{\lambda_{p}+2 \sqrt{\lambda_{p} \lambda_{b}}}+\frac{1}{4 \sqrt{\lambda_{p} \lambda_{b}}}\right\} .
$$

It is worthwhile to note that the resulting expression for $\chi_{\text {imp }}$ is proportional to $\epsilon$ and not to $\epsilon^{2}$ as is apparent from (2.29), because of the partial cancellation caused by $\epsilon$-dependence of the thermal masses (2.34).

\section{The Knight shift}

The magnetization response to a uniform magnetic field is space-dependent due to the presence of an impu- 
rity; experimentally, this can be detected in NMR experiments by measuring the Knight shift. It is not difficult to generalize the calculation of the previous subsection to the space-dependent case; the "uniform" (slowly varying on the scale of a lattice constant) component of the Knight shift $K_{u}(x)$ is given by

$$
K_{u}(x)=\int d^{d} x^{\prime} \chi_{u}\left(x, x^{\prime}\right)
$$

where $\chi_{u}\left(x, x^{\prime}\right)$ is a generalization of (2.28),

$$
\chi_{u}\left(x, x^{\prime}\right)=\frac{T}{N^{2}-1} \sum_{a} \frac{\delta^{2} \ln \mathcal{Z}}{\delta H_{u a}(x) \delta H_{u a}\left(x^{\prime}\right)} .
$$

This uniform Knight shift obeys the scaling form in Eq. (1.4). A direct application of Eq. (2.22) to this scaling form implies, as in Ref. 9 that in the quantum-critical region

$$
K_{u}(x \rightarrow 0) \sim \frac{1}{T^{1-\eta^{\prime} / 2}} \frac{1}{|x|^{d-\eta^{\prime} / 2}}
$$

One can see that $\chi_{u}\left(x, x^{\prime}\right)$ is determined by the same set of diagrams of Fig. 31(a-g) but with momenta flowing into the external vertices. To obtain the Knight shift from Eq. (2.38), it is useful to define the Fourier transform $\chi_{u}(q)$ by

$$
K_{u}(x)=\int \frac{d^{d} q}{(2 \pi)^{d}} \chi_{u}(q) e^{i q x}
$$

and then the diagrams have momentum $q$ flowing out of one external vertex. Obviously, $\chi_{\mathrm{imp}}=\chi_{u}(q=0)$ and the normalization (1.5) is satisfied. Similarly to the case of susceptibility considered above, one can show that the Knight shift is identically zero at $T=0$. At finite temperature the main contribution to $\chi_{u}(q)$ is given by the diagrams of Fig. 3(a,b) with the Matsubara frequency in each propagator replaced by the corresponding thermal mass (2.34), yielding

$$
\begin{aligned}
\chi_{u}(q) & \simeq 2 Q^{2}\left(e_{0}^{2}\right)^{2} \widetilde{S} T\left\{I\left(q, m_{p}\right) I\left(q, m_{b}\right)\right. \\
& \left.+2 \int \frac{d^{d} k}{(2 \pi)^{d}} \frac{I\left(k, m_{b}\right)}{\left(k^{2}+m_{p}^{2}\right)\left[(k-q)^{2}+m_{p}^{2}\right]}\right\}
\end{aligned}
$$

where we have denoted

$$
I(q, m)=\int \frac{d^{d} k}{(2 \pi)^{d}} \frac{1}{\left(k^{2}+m^{2}\right)\left[(k+q)^{2}+m^{2}\right]} .
$$

We will only need the expression for $I(q, m)$ in $d=3$ :

$$
I_{d=3}(q, m)=\frac{1}{4 \pi|q|} \arctan \frac{|q|}{2 m} .
$$

Asymptotic expressions for $\chi_{u}(q)$ can be easily obtained. For small wave vectors $q \ll 2 m_{b}$ one recovers the results for the impurity susceptibility from the previous subsection, $\chi_{u}\left(q \ll 2 m_{b}\right) \simeq \chi_{\mathrm{imp}}$, while for large wave vectors $q \gg m_{p}$ one has

$$
\chi_{u}(q) \simeq \widetilde{S} T\left(\frac{12 \pi Q \epsilon}{N}\right)^{2} \frac{\ln \left(q / m_{p}\right)}{q^{2}}, \quad q \gg m_{p} .
$$

At large distances $r \gg m_{p}^{-1}$ the Knight shift decays exponentially as $e^{-m_{p} r}$ due to the finite photon mass $m_{p}$. At small distances the Knight shift has the following behavior:

$$
K_{u}(r) \simeq 36 \pi \widetilde{S} T\left(\frac{Q \epsilon}{N}\right)^{2} \frac{1}{r} \ln \left(\frac{1}{m_{p} r}\right), \quad m_{p} r \ll 1 .
$$

Notice that $m_{p}^{2} \propto \epsilon T^{2}$, so at low temperature the condition $m_{p} r \ll 1$ will be valid for a wide range of $r$. Apart from the log correction, (2.45) is consistent with the general scaling forms (1.4), (2.22), and (2.40); the logarithm is likely an artifact of the integer-valued exponents that appear at low orders in the computation.

In a similar manner, one can calculate the staggered Knight shift

$$
K_{s}(x)=\frac{T}{N^{2}-1} \sum_{a} \frac{\delta^{2} \ln \mathcal{Z}}{\delta H_{u a} \delta H_{s a}(x)} .
$$

This obeys a scaling form analogous to Eq. (1.4)

$$
K_{s}(x)=\frac{T^{(D-2+\eta) / 2}}{T} \Phi_{s}(T x)
$$

where the behavior as $x \rightarrow 0$ analogous to Eq. (2.40) is

$$
K_{s}(x \rightarrow 0) \sim \frac{1}{T^{1-\eta^{\prime} / 2}} \frac{1}{|x|^{-\Delta_{\mathrm{imp}}^{\phi}}} .
$$

The staggered Knight shift is computed by the Fourier transform of the momentum-dependent staggered susceptibility $\chi_{s}(q)$ which is in the leading order determined by the diagrams of Fig. 3/h,i), with an uncompensated momentum $q$ being "injected" in the staggered magnetic field vertex of Fig. 2(d). Notice that for the staggered susceptibility the first nonvanishing contribution appears already in the first order in the impurity charge $Q$. As in the uniform case, the staggered susceptibility can be proven to be identically zero at $T=0$, and at finite temperature the result is again determined by the zero Matsubara frequency term and thus by the diagram Fig. 3) i), with the zero frequency replaced in each propagator by the respective thermal mass. This yields

$$
\chi_{s}(q)=\frac{48 \epsilon \pi^{2} Q \widetilde{S} T}{N} \frac{I\left(q, m_{b}\right)}{q^{2}+m_{p}^{2}} .
$$

Using (2.43), one can easily deduce the asymptotic behavior of the staggered Knight shift at small distances $r \ll m_{p}^{-1}$ :

$$
K_{s}(r)=\frac{3 \epsilon Q \widetilde{S} T}{N} \ln \left(\frac{1}{m_{p} r}\right), \quad m_{p} r \ll 1 .
$$


Again, apart from the logarithmic correction, this result is in accord with the scaling form suggested by (2.21) and (2.48). It is worthwhile to note that in $\mathrm{CP}^{N-1}$ model for small $\epsilon=4-D$ and large $N$ the staggered magnetization response $K_{s}(x)$ to an external magnetic field is much stronger than the uniform response $K_{u}(x)$.

\section{Néel ordered phase}

In the Néel phase, one of the components of the $z$ field gets condensed. Assuming $\left\langle z_{\alpha}\right\rangle \propto \delta_{\alpha 1}$, we can parameterize

$$
z_{\alpha}=\left\{\begin{array}{cc}
(\sqrt{|s| / u}+\sigma) e^{i \zeta} & \text { for } \alpha=1 \\
\psi_{\alpha} e^{i \zeta} & \text { for } \alpha>1
\end{array}\right.
$$

where $\sigma$ is a real field, and $\psi_{\alpha}, \alpha=2 \ldots N$, are $N-1$ complex fields. The overall phase $\zeta$ can be gauged away, and we can set $\zeta=1$. Then $A_{\mu}$ becomes a massive gauge boson, with the "mass" $\sqrt{2 e_{0}^{2}|s| / u}$ acquired by the Higgs mechanism. Inserting (2.51) in $\mathcal{S}_{b}+\mathcal{S}_{\text {imp }}$ we can obtain a straightforward perturbative expansion for all physical properties in powers of $u_{0}$ and $e_{0}^{2}$.

First we describe the behavior of the Néel order near the impurity. We expect this to obey the scaling form

$$
\left\langle\phi_{a}(x)\right\rangle=|s|^{\beta} F_{\phi}\left(x /|s|^{\nu}\right)
$$

where $\beta=(D-2+\eta) \nu / 2$. For large $x$, we expect that $F_{\phi}$ approaches a constant which characterizes the bulk order, while for small $x$ near the impurity we may deduce from (2.22) that

$$
\lim _{y \rightarrow 0} F_{\phi}(y) \sim \frac{1}{y^{\left(D-2+\eta-\eta^{\prime}\right) / 2}}
$$

Explicitly, computing $\left\langle\phi_{a}\right\rangle$ by the perturbative expansion defined above, we find that there are no impurity dependent corrections at first order beyond tree level. So to this order, $F_{\phi}(y)$ is independent of $y$, but $y$ dependent terms do appear at higher orders in $\epsilon$. The spatially independent response at this order is also consistent with the exponent relation (2.19) and 2.53).

Turning next to the uniform magnetization, we now expect from the scaling relations in Section $\amalg B$ that

$$
\left\langle M_{a}(x)\right\rangle=|s|^{d \nu} F_{m}\left(x /|s|^{\nu}\right)
$$

where now the scaling function obeys

$$
\lim _{y \rightarrow 0} F_{m}(y) \sim \frac{1}{y^{d-\eta^{\prime} / 2}}
$$

It is easy to compute $M_{a}$ to one-loop order, and we find

$$
\left\langle M_{a}(x)\right\rangle=\frac{Q T_{11}^{a} 2 e_{0}^{2}|s|}{u} \int \frac{d^{d} q}{(2 \pi)^{d}} \frac{e^{i q x}}{q^{2}+2|s| e_{0}^{2} / u}
$$

This obeys the scaling form in (2.54) with a magnetization that decays exponentially on a scale set by the "photon" mass. For small $x$, note that to leading order in $\epsilon,\left\langle M_{a}(x)\right\rangle \sim 1 / x$, and this is consistent with (2.55) and the value of $\eta^{\prime}$.

An interesting property of (2.56) is that the total magnetization near the impurity is given by

$$
\int d^{d} x\left\langle M_{a}(x)\right\rangle=Q T_{11}^{a} .
$$

As in Ref. 9, this relation is expected to be exact, and the total magnetization near the impurity is therefore quantized. The quantization is a consequence of the conservation of total spin. Here it may be viewed as an interesting consequence of Meissner flux expulsion induced by the condensation of the $z_{\alpha}$. In the presence of an applied field $H_{a}$, the fact that the $z_{\alpha}$ condensate is polarized along the $\alpha=1$ direction, the Meissner effect implies from (2.14) that $i A_{\tau}+H_{a} T_{11}^{a}$ will fluctuate around zero. So setting $A_{\tau}=i H_{a} T_{11}^{a}$, we obtain from (1.2) a term in the effective action which is linear in $H_{a}$, and whose coefficient is the total magnetization in (2.57).

\section{E. Spin gap phase}

Finally, we turn to the spin gap phase which appears for sufficiently large $s$. We will not include 'dangerously irrelevant' monopole effects here $\frac{19,20.21}{1}$ which are believed to lead to VBS order and confinement at the longest length scales. We will restrict our attention to length scales of order $1 / \Delta$, where $\Delta$ is the spin gap. We will argue that in $d=2$ a spinon is necessarily bound to the impurity, and so the onset of confinement at longer scales does not have a significant influence on the behavior of the impurity.

It is straightforward to extend the $\epsilon$ expansion to the spin gap phase. The spinons experience the Coulomb potential of the impurity $\sim 1 / r^{d-2}$. This potential may form bound state, but because of the bulk spin gap, these states are above the ground state. So the spin gap is preserved in the presence of the impurity and all magnetic response functions vanish exponentially at low temperature.

We now argue that these conclusions of the $\epsilon$ expansion are strongly modified in $d=2$. The key point is that the Coulomb potential $\sim \ln (r)$ in $d=2$, and this increases without bound as $r \rightarrow \infty$. Consequently the self energy of a naked impurity with nonzero charge diverges logarithmically with system size. Therefore, it always pays to create a spinon above the spin gap, and bind it to the impurity, and neutralize its charge. The scale at which this occurs can be estimated using the $1 / N$ expansion. ${ }^{43}$ In this expansion, the Coulomb interaction is screened by the vacuum polarization of spin-anti-spinon pairs to a value of order $(Q \Delta / N) \ln (R \Delta)$. The spinons obey a Schrö dinger equation in this potential with a mass $\Delta$, and from this we obtain an estimate for the size of the bound state of order $R \sim(N / Q)^{1 / 2} / \Delta$. At length scales larger than this $R$, the impurity will just appear as an 
isolated spin moment, and will therefore contribute the ordinary Curie susceptibility $=1 /(4 T)$ (for a $Q= \pm 1$ impurity).

Thus the impurity simply behaves as a free local moment in the spin gap phase. Note that we did not have to appeal to confinement physics to reach this conclusion; confinement, and VBS order, appears at the parametrically larger scale ${ }^{43}$ of order $a(a / \Delta)^{\tilde{\alpha} N}$, where $a$ is the short-distance cutoff.

\section{STAGGERED FLUX SPIN LIQUID}

This section will examine the response of a second algebraic spin liquid to impurities: the staggered flux spin liquid with fermionic spinon excitations ${ }^{35.36 .37 .38}$ We will see that the scaling structure is rather similar to that of the critical $\mathrm{CP}^{N-1}$ model examined in Section [II] although there will be some crucial differences in some physical properties.

The low energy excitations of the spin liquid are described by $N_{f}$ flavors of 2-component Dirac fermions, $\Psi$, coupled to the $\mathrm{U}(1)$ gauge field $A_{\mu}$ with the action

$$
\mathcal{S}_{b}=\int d^{3} y \bar{\Psi}\left[-i \gamma^{\mu}\left(\partial_{\mu}+i A_{\mu}\right)\right] \Psi .
$$

As before, $y=(\tau, \vec{x})$ is the spacetime coordinate, and $\mu$ extends over the $D=3$ spacetime indices. The Dirac matrices $\gamma^{\mu}=\left(\tau^{3}, \tau^{2},-\tau^{1}\right)$, where $\tau^{\mu}$ are the Pauli matrices, and the field $\bar{\Psi}=i \Psi^{\dagger} \tau^{3}$ - we follow the notation of Hermele et al $b^{37}$ The number of flavors is $N_{f}=4$ for the staggered flux state, and our results are also extended to the so-called $\pi$-flux state, which has $N_{f}=8$. Our analysis will be carried out, as in the previous works, in a $1 / N_{f}$ expansion. We have not included a bare Maxwell term for the gauge field in $\mathcal{S}_{b}$ because it turns out to be irrelevant at all orders in the $1 / N_{f}$ expansion.

\section{A. Bulk theory}

The structure of the bulk theory has been described in some detail in Refs. 35. 37, and we will not repeat the results here. In the large $N_{f}$ limit, the propagator of the gauge field in the Lorentz gauge is

$$
D_{\mu \nu}(p)=\left(\delta_{\mu \nu}-\frac{p_{\mu} p_{\nu}}{p^{2}}\right) \frac{16}{N_{f} p}
$$

This propagator arises from the vacuum polarization of the fermions. Notice that it is suppressed by a power of $1 / N_{f}$, so the $1 / N_{f}$ expansion can be setup as a perturbation theory in the fermion-gauge field interaction.

A large number of order parameters can be constructed out of fermion bilinears, and a detailed catalog has been presented ${ }^{37}$ Among these are the $\mathrm{SU}\left(N_{f}\right)$ flavor currents

$$
J_{\mu}^{a}=-i \bar{\Psi} \gamma^{\mu} T^{a} \Psi
$$

certain components of which are the magnetization density and current. Conservation of this current implies the scaling dimension

$$
\operatorname{dim}\left[J_{\mu}^{a}\right]=2,
$$

which is the analog of the relation (2.16) in $d=2$ (we will restrict all discussion in this section to $d=2$ ).

Also considered were the following quantities:

$$
N^{a}=-i \bar{\Psi} T^{a} \Psi, \quad M=-i \bar{\Psi} \Psi,
$$

which relate to additional order parameters including the Néel order (note that $M$ does not correspond to the physical magnetization). Their scaling dimensions have been computed in the $1 / N_{f}$ expansion:

$$
\operatorname{dim}\left[N^{a}\right]=2-\frac{64}{3 \pi^{2} N_{f}}+\mathcal{O}\left(1 / N_{f}^{2}\right)
$$

\section{B. Relationship to earlier work}

Before describing the results of our analysis of the impurity in the $\mathrm{U}(1) \mathrm{sF}$ phase, it is useful to describe the earlier analyses in Refs. 24 25 262728. They ignored the $A_{\mu}$ fluctuations, but instead considered a theory of fermionic spinons $f_{i \alpha}$ on the sites, $i$, of the square lattice; $\alpha$ is a spin index. These spinons obey $\sum_{\alpha}\left\langle f_{i \alpha}^{\dagger} f_{i \alpha}\right\rangle=1$ on every lattice site except at the impurity $i=0$. Here, they inserted a vacancy (representing e.g. a Zn impurity) by including a potential term in the Hamiltonian

$$
\mathcal{H}_{\text {imp }}=V \sum_{\alpha} f_{0 \alpha}^{\dagger} f_{0 \alpha}
$$

and taking the limit $V \rightarrow \infty$ to prohibit any spinons from residing on the vacancy. The key physical ingredient in these analyses is the difference in the spinon occupation number between the impurity and the bulk:

$$
\sum_{\alpha}\left\langle f_{i \alpha}^{\dagger} f_{i \alpha}\right\rangle-1=-\delta_{i 0}
$$

In our approach the analog of the $V=\infty$ limit is obtained by the functional integral over $A_{\tau}$. In the bulk theory, the continuum field $\Psi$ is defined so that $\left\langle\Psi^{\dagger} \Psi\right\rangle=0$ in the absence of the impurity, and the continuum analog of Eq. (3.8) is

$$
\left\langle\Psi^{\dagger} \Psi(r)\right\rangle=-Q \delta^{2}(r)
$$

where the rhs has a Dirac delta function. The constraint in Eq. (3.9) is imposed not by adding a potential energy, but by the functional integral over $A_{\tau}$, which appears in the Lagrangian density of $\mathcal{S}_{b}+\mathcal{S}_{\text {imp }}$ (with $\mathcal{S}_{b}$ given in Eq. (3.1) ) as $i A_{\tau}\left(\Psi^{\dagger} \Psi+Q \delta^{2}(r)\right)$. Our treatment of gauge fluctuations ensures that the constraint on the spinon occupations is imposed not just on the average, but dynamically on all states and on all sites. The equality in 


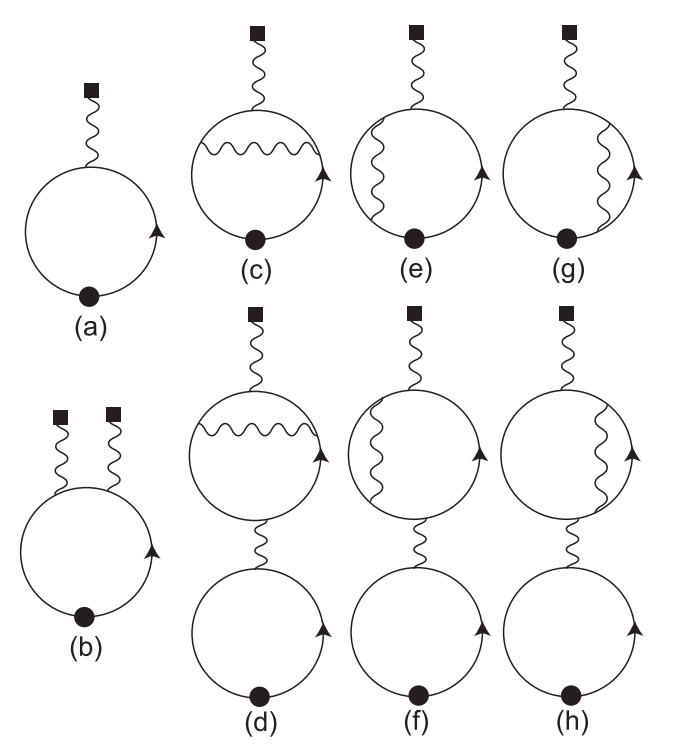

FIG. 5: Feynman diagrams to order $1 / N_{f}$ for the lhs of Eq. (3.9). The full line is the Dirac fermion propagator, the wave line is the photon propagator, the filled square is the impurity source term in $\mathcal{S}_{\text {imp }}$, and the filled circle is $\Psi^{\dagger} \Psi$ vertex. As noted in the text, the diagram in (b) has an odd number of photon vertices, and so vanishes by Furry's theorem ${ }^{44}$ Here, and henceforth, we do not show a number of other diagrams which vanish because of Furry's theorem.

Eq. (3.9) holds to all orders in the $1 / N_{f}$ expansion: this follows from the 'equation of motion' for $A_{\tau}$

$$
\left\langle\frac{\delta}{\delta A_{\tau}}\left(\mathcal{S}_{b}+\mathcal{S}_{\text {imp }}\right)\right\rangle=0 .
$$

It is instructive to also test Eq. (3.9) by explicitly evaluating the lhs of Eq. (3.9) in the $1 / N_{f}$ expansion. The corresponding Feynman diagrams are shown in Fig. 5 At leading order, we have the diagram Fig [5(a), which is easily evaluated to yield the rhs of Eq. (3.9). At order $1 / N_{f}$, the diagrams shown in Fig. 5 (b-g) contribute. Of these, Fig. (b) vanishes by Furry's theorem. ${ }^{44}$ Of the remaining, it is easy to show that they cancel in pairs: this requires only the knowledge that the photon propagator is the inverse of the fermion vacuum polarization bubble.

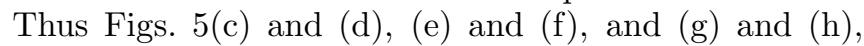
all cancel against each other, and Eq. (3.9) is thus established to this order. It is not difficult to extend these arguments to all orders in $1 / N_{f}$. Note that it is possible to satisfy Eq. (3.9) in a perturbative treatment of gauge fluctuations, without the need to appeal to bound states: the delta function at $r=0$ arises from a superposition of the contribution of many extended states, rather than from a bound state claimed earlier 24.25 .26

It is clear that our approach yields a systematic and controlled treatment of the spinon deficit at the impurity, in contrast to the earlier ad-hoc mean-field approaches 24.25 .26 .27 .28 Our analysis implies that the Curie constant $\mathcal{C}$ is a non-trivial number, with contributions at all orders in $1 / N_{f}$, and is not given simply by the Curie constant of a single spin.

The proper analysis of a potential term like that in Eq. (3.7) requires the scaling analysis of perturbations to the conformal field theory defined by $\mathcal{S}_{b}+\mathcal{S}_{\text {imp }}$. The action for such a perturbation takes the form

$$
\mathcal{S}_{b}^{\prime}=V \int d \tau \Psi^{\dagger} \Psi(x=0, \tau)
$$

A simple analysis of scaling dimensions shows that

$$
\operatorname{dim}[V]=-1+\mathcal{O}\left(1 / N_{f}\right) .
$$

So, potential scattering is an irrelevant perturbation at all orders in the $1 / N_{f}$ expansion.

A further distinction between our results and the earlier work ${ }^{24.25 .26 .27 .28}$ appears in the response to a uniform magnetic field. This has significant experimental consequences, and will be discussed in Section IIID

\section{Impurity exponents}

The analysis is analogous to that carried out in Section $\llbracket \mathrm{B}$ for the $\mathrm{CP}^{N-1}$ model. As there, and for similar reasons, we neglect the possible influence of monopoles.

Here, we will determine the impurity renormalization to the order $1 / N_{f}^{2}$. To this order, we find that all the operators introduced above, the $J_{\mu}^{a}$ and $N^{a}$ acquire a common correction, $\Delta_{\text {imp }}$, to their bulk scaling dimension. This correction is given by the single diagram in Fig 1(c), which (along with its symmetry related partner) evaluates to

$$
\begin{aligned}
& \frac{512 Q^{2}}{N_{f}^{2}} \int \frac{d^{2} q}{(2 \pi)^{2}} \frac{d^{2} k}{(2 \pi)^{2}} \frac{(-i \omega+\vec{q} \cdot \vec{\tau})(-i \omega+(\vec{k}+\vec{q}) \cdot \vec{\tau})}{k q\left(q^{2}+\omega^{2}\right)\left((q+k)^{2}+\omega^{2}\right)} \\
& \quad=\frac{128 Q^{2}}{N_{f}^{2} \pi^{2}} \ln (\Lambda / \omega)
\end{aligned}
$$

where $\vec{\tau}=\left(\tau^{1}, \tau^{2}\right)$ and $\Lambda$ is an ultraviolet cutoff; here and henceforth we are using the fermion lines to represent the propagator $(i \omega+\vec{\tau} \cdot \vec{q})^{-1}$ from $\Psi$ to $\Psi^{\dagger}$ (rather then the Dirac propagator from $\Psi$ to $\bar{\Psi}$ ). There are a number of other diagrams, like those shown in Fig. [1 which could contribute to the vertex renormalization; however they do not contribute either for reasons as discussed for the $\mathrm{CP}^{N-1}$ model, or because of Furry's theorem. From this we obtain the impurity correction to the scaling dimension

$$
\Delta_{\mathrm{imp}}=-\frac{128 Q^{2}}{N_{f}^{2} \pi^{2}}+\mathcal{O}\left(1 / N_{f}^{3}\right)
$$

We expect that the higher order corrections will not be the same for the $J_{\mu}^{a}$ and $N^{a}$. 

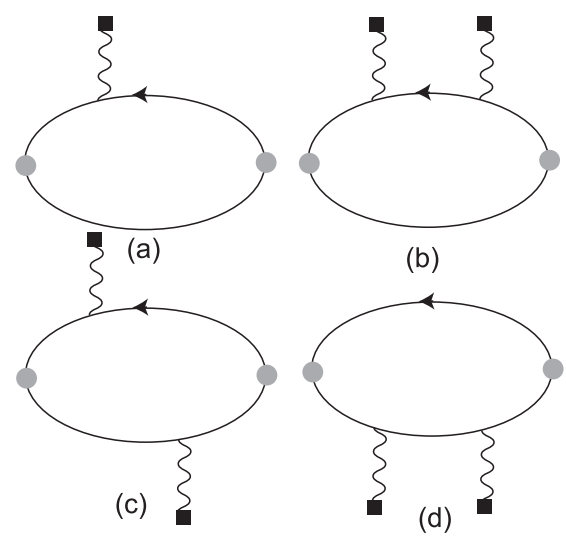

(b)

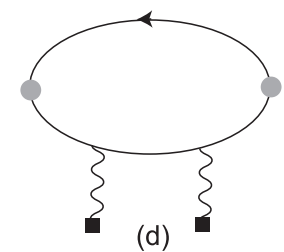

FIG. 6: Feynman diagrams for the impurity susceptibility and Knight shifts of the staggered flux spin liquid. The grey circle vertices depend upon the correlator being evaluated: they equal $(i) T^{a} \gamma_{\tau}$ for the ferromagnetic spin density $J_{\tau}^{a}$, (ii) $T^{a}$ for the order parameter $N^{a}$, and (iii) unity for $M$.

\section{Linear response to a uniform applied field}

Again, we proceed in analogy to the analysis in Section ПC We apply a uniform magnetic field $H_{a}$, associated with the $\mathrm{SU}\left(N_{f}\right)$ generator $T^{a}$, which couples linearly to the conserved total spin density $J_{\tau}^{a}$. In principle, in the presence of the impurity, the linear response to this applied field can induce time-independent, spacedependent average values of not only the spin density, $J_{\tau}^{a}$, but also of the other order parameters $M$, and $N^{a}$. This would be analogous to the non-zero averages of the uniform and staggered magnetizations induced by an applied magnetic field on the $\mathrm{CP}^{N-1}$ model (which led to the uniform and staggered Knight shifts). However, here we will find a crucial difference. In the scaling limit of the algebraic spin liquid represented by Eq. (3.1), $H_{a}$ induces only a non-zero $J_{\tau}^{a}$, and average values of all the $N^{a}$ and $M$ are zero. Thus there is only a uniform Knight shift, $K_{u}(x)$, and all the 'staggered' Knight shifts, $K_{s}(x)$, associated with the many competing orders are zero. The staggered Knight shift can appear only if some corrections to scaling are included, associated with irrelevant operators which reduce the symmetry of the conformal theory to that of the lattice model, and so can be expected to be weaker than the uniform Knight shift.

The vanishing of $\langle M(x)\rangle$ and $\left\langle N^{a}(x)\right\rangle$ in the presence of $H_{a}$ can be established by a careful consideration of the symmetries of the Dirac fermion theory. First $\langle M\rangle=$ 0 , to linear order in $H_{a}$, simply by $\mathrm{SU}\left(N_{f}\right)$ symmetry. Establishing the value of $\left\langle N^{a}\right\rangle$ requires more complicated considerations. Let us consider the leading contribution to $\left\langle N^{a}\right\rangle$, to linear order in $H_{a}$, in the $1 / N_{f}$ expansion, represented by the graph in Fig 6/a). The value of the fermion loop is proportional to

$$
\begin{aligned}
& T \sum_{\omega_{n}} \int \frac{d^{2} k}{4 \pi^{2}} \operatorname{Tr}\left[\left(i \omega_{n}+\vec{\tau} \cdot \vec{k}\right)^{-1} \tau^{3}\left(i \omega_{n}+\vec{\tau} \cdot \vec{k}\right)^{-1}\right. \\
& \left.\quad \times\left(i \omega_{n}+\vec{\tau} \cdot(\vec{k}+\vec{q})\right)^{-1}\right]
\end{aligned}
$$

Evaluating the trace over the Dirac matrices, we obtain an identical zero. This can be understood as a consequence of time-reversal invariance. Both $H_{a}$ and $N^{a}$ are odd under the time reversal, 36 as is the charge of the impurity $Q$. So an expansion of $\left\langle N^{a}\right\rangle / H_{a}$ can only involve even powers of $Q$. Proceeding to order $1 / N_{f}^{2}$, we obtain diagrams like those shown in Fig. 6] (b-d), which have a pre-factor of $Q^{2}$, and so are potentially non-zero. However, these diagrams have a fermion loop with an odd number of $\gamma^{\mu}$ vertices, and so vanish by Furry's theorem. 44 By a combination of Furry's theorem and time-reversal invariance we can now easily see that all terms vanish and so $\left\langle N^{a}\right\rangle=0$. Because of the $T^{a}$ matrices in the definitions of $N^{a}$ and $J_{\tau}^{a}$, there must be a single fermion loop which connects the external vertices. By Furry's theorem, this loop must have an odd number of photon vertices. All other fermion loops can only have an even number of photon vertices. Consequently, there must be an odd number of photon vertices remaining to connect to the external impurity source term. However, by time-reversal, there must be an even number of impurity terms; hence the result.

Our conclusion that $\left\langle N^{a}\right\rangle=0$ is starkly different from the mean-field prediction 24.25 .26 .27 .28 of an induced moment which oscillated strongly between the two square sublattices. Such oscillations can only appear upon including irrelevant operators.

It remains only to compute the uniform Knight shift $K_{u}(x)$, or equivalently, its Fourier transform $\chi_{u}(q)$ defined in Eq. (2.41). The scaling analysis of Section IIC implies that this Knight shift obeys the scaling form in Eq. (1.4), with the $x \rightarrow 0$ behavior given by

$$
K_{u}(x \rightarrow 0) \sim T^{d-1+\Delta_{\mathrm{imp}}} \frac{1}{|x|^{-\Delta_{\mathrm{imp}}}}
$$

Here we expect that the $\Delta_{\text {imp }}$ exponent is that associated with $J_{\tau}^{a}$, and not (unlike the situation with the $\mathrm{CP}^{N-1}$ model) that associated with the $N^{a}$, because there is no 'mixing' between the staggered and uniform magnetizations near the impurity for the $\mathrm{sF}$ spin liquid.

At first order in $1 / N_{f}, \chi_{u}(q)$ is given by the diagram in Fig[6(a), which vanishes by Furry's theorem. The leading non-vanishing contribution is at order $1 / N_{f}^{2}$, and is given by the diagrams in Fig 6 (b-d) (a number of order $1 / N_{f}^{2}$ diagrams which vanish because of Furry's theorem are not shown). The sum of these diagrams can we written 
in the following compact form

$$
\begin{aligned}
\chi_{u}(q)= & Q^{2} \widetilde{S} T \sum_{\omega_{n}} \int \frac{d^{2} k}{4 \pi^{2}} \frac{d^{2} p}{4 \pi^{2}} \frac{\partial}{\partial\left(i \omega_{n}\right)} \operatorname{Tr}\left[\left(i \omega_{n}+\vec{\tau} \cdot \vec{k}\right)\right. \\
\times & \left.\left(i \omega_{n}+\vec{\tau} \cdot(\vec{k}+\vec{q})\right)\left(i \omega_{n}+\vec{\tau} \cdot(\vec{k}+\vec{p})\right)\right]^{-1} \\
& \times D_{\tau \tau}(p) D_{\tau \tau}(|\vec{q}-\vec{p}|) .
\end{aligned}
$$

Here $D_{\tau \tau}$ is the $\tau$ component of the photon propagator, representing the external lines connecting to the impurities, and $\widetilde{S}$ is the constant proportional to the $\mathrm{SU}(\mathrm{N})$ Casimir eigenvalue as defined in (2.30). Notice that the expression is a total frequency derivative; this immediately implies that $\chi_{u}(q)=0$ at $T=0$, when the frequency summation can be converted to an integration. This vanishing is a consequence of the conservation of total spin.

A non-zero $\chi_{u}(q)$ is obtained at $T>0$, and we now compute this. First we need the photon propagator at $T>0$. This is given by a single fermion loop and at this order we only need the $\tau, \tau$ component at a spatial momentum $\vec{q}$ :

$$
\begin{array}{rl}
D_{\tau \tau}^{-1}(q)=-N_{f} & T \sum_{\omega_{n}} \int \frac{d^{2} k}{4 \pi^{2}} \operatorname{Tr}\left[\left(i \omega_{n}+\vec{\tau} \cdot \vec{k}\right)\right. \\
\left.\times\left(i \omega_{n}+\vec{\tau} \cdot(\vec{k}+\vec{q})\right)\right]^{-1}
\end{array}
$$

We first combine the denominators in Eq. (3.18) using the Feynman parameter $u$, perform the frequency summation, and finally integrate over $k$. This yields

$$
D_{\tau \tau}^{-1}(q)=\frac{N_{f} T}{\pi} \int_{0}^{1} d u \ln \left[2 \cosh \left(\frac{q}{2 T} \sqrt{u(1-u)}\right)\right] .
$$

Eq. (3.19) interpolates between $N_{f} q / 16$ for $T \ll q$ which agrees with the $T=0$ result in Eq. (3.2), to $(T / \pi) \ln 2$ for $q \ll T$.

Inserting Eq. (3.19) into Eq. (3.17), we conclude that $\chi_{u}(q)$ obeys the scaling form

$$
\chi_{u}(q)=\frac{1}{T} \widetilde{\Phi}_{u}(q / T)
$$

where the scaling function $\widetilde{\Phi}_{u}$ is the Fourier transform of the scaling function $\Phi_{u}$ in Eq. (1.4). Further, $\mathcal{C}$, the anomalous Curie constant appearing in Eq. (1.3), equals $\widetilde{\Phi}_{u}(0)$. To determine the scaling function $\widetilde{\Phi}_{u}$, we need to evaluate Eq. (3.17). We combined the Green's functions using two Feynman parameters $u$, $v$, evaluated the integral over $k$, differentiated with respect to frequency, and

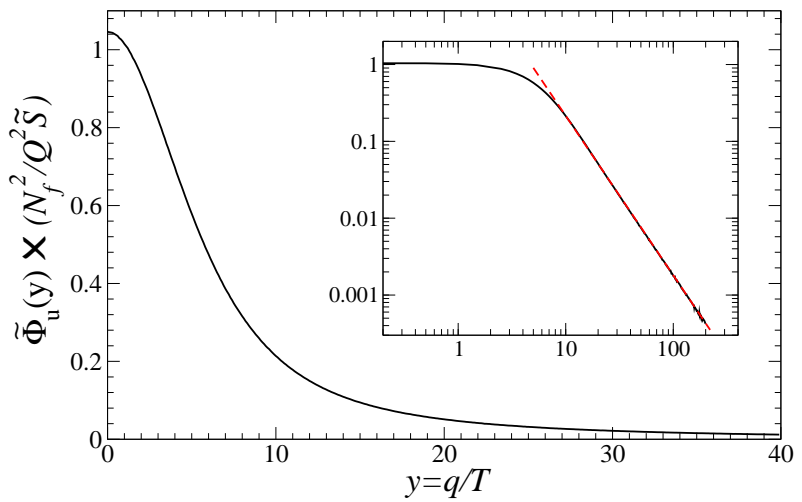

FIG. 7: Numerically calculated scaling function $\widetilde{\Phi}_{u}(y)$ defined by (3.19 3.23). The inset shows large- $y$ behavior together with the least square fit (dashed line) to a power law for $y>40$ : the fit yields $\widetilde{\Phi}_{u}(y) \simeq \mathcal{C}^{\prime} / y^{\alpha}$ with $\mathcal{C}^{\prime}=24.7(1)$ and $\alpha=2.070(5)$.

finally evaluated the summation over $\omega_{n}$. This yields

$$
\begin{aligned}
\chi_{u}(q)= & -\frac{Q^{2} \widetilde{S}}{\pi} \int_{0}^{1} d u \int_{0}^{1-u} d v \int \frac{d^{2} p}{4 \pi^{2}}\left\{f^{\prime}(\Delta)\right. \\
+ & {\left[2 \Delta^{2}-u q^{2}-v p^{2}-(1-2(u+v)) \vec{p} \cdot \vec{q}\right] } \\
& \left.\times \frac{f^{\prime \prime}(\Delta)}{4 \Delta}\right\} D_{\tau \tau}(p) D_{\tau \tau}(|\vec{q}-\vec{p}|)
\end{aligned}
$$

where

$$
\Delta^{2}=q^{2} u(1-u)+p^{2} v(1-v)-2 u v \vec{p} \cdot \vec{q}
$$

and $f$ is the Fermi function

$$
f(\varepsilon)=1 /\left(e^{\varepsilon / T}+1\right) .
$$

The remaining integrals have to be evaluated numerically. From such an evaluation at $q=0$ we found

$$
\mathcal{C}=\frac{1.0460(5) \widetilde{S} Q^{2}}{N_{f}^{2}}+\mathcal{O}\left(1 / N_{f}^{3}\right)
$$

for the universal Curie constant appearing in Eq. (1.3). The calculated shape of the scaling function $\widetilde{\Phi}_{u}(y)$ is shown in Fig. 7 From the numerical analysis of its behavior for large arguments, we deduce that $\widetilde{\Phi}_{u}(y)$ has a power-law decay $\widetilde{\Phi}_{u}(y) \propto 1 / y^{\alpha}$ at $y \gg 1$, where the exponent $\alpha \approx 2$ within the accuracy we were able to achieve when calculating the four-dimensional integral in (3.21). Therefore, we obtain that at small distances the uniform Knight shift $K_{u}(x)$ tends to a (lattice cutoff-dependent) constant as $x \rightarrow 0$, which is consistent, to the leading order in $1 / N_{f}$, with Eq. (3.16) and the fact that $\Delta_{\text {imp }}$ vanishes at this order (see Eq. (3.14)). 


\section{OTHER SPIN LIQUIDS}

This section will discuss the extension of our results to some other examples of spin liquids. The cases considered below do not share the high degree of universality found in the critical $\mathrm{CP}^{N-1}$ and $\mathrm{sF}$ spin liquids above: some aspects depends upon microscopic details. We will consider the spinon Fermi surface in Section IVA and $Z_{2}$ spin liquids in Section IVB

\section{A. Spinon Fermi surface}

This spin liquid differs from the sF spin liquid by having a Fermi surface of gapless spinon excitations, with a non-zero density of states. The bulk theory is not a CFT, and the bulk action for the fermionic spinons $f_{\alpha}$ is

$$
\begin{array}{r}
\mathcal{S}_{b}=\int d \tau \int \frac{d^{d} k}{(2 \pi)^{d}}\left[f _ { \alpha } ^ { \dagger } ( k , \tau ) \left(\frac{\partial}{\partial \tau}+i A_{\tau}\right.\right. \\
+\epsilon(\vec{k}-\vec{A}))] f_{\alpha}(k, \tau)
\end{array}
$$

Here $\alpha$ is a $\operatorname{SU}(N)$ spinor index, $\epsilon(\vec{k})$ is the spinon dispersion, and the Fermi surface defined by $\epsilon(\vec{k})=0$ has a finite density of zero energy spinon excitations. The properties of this spin liquid can be computed by a combinations of previous methods $\frac{45,46}{}$ and the methods introduced here.

In the absence of the impurity, the spin susceptibility is finite as $T \rightarrow 0$, equal to the density of states at the Fermi level. The impurity introduces a 'Coulomb' potential for the spinons. In the small momentum limit, $k \rightarrow 0$, this interaction is screened to a constant value by the Fermi surface excitations. Evaluating the response to an applied magnetic field using a diagram like that in Fig. 6]a), we conclude that $\chi_{\mathrm{imp}}(T \rightarrow 0)$ is finite and proportional to the energy derivative of the density of states at the Fermi level.

Upon considering the spatial dependence of the Knight shift, again using the diagram in Fig. 6(a), we find that the dominant response oscillates at the ' $2 k_{F}$ ' wavevector 45 The transverse gauge interactions strongly enhance the impurity-spinon vertex, and this leads to a singular response at $2 k_{F}$ decaying with a power-law of $x$ determined in Refs. 45 46. Again, this response is finite as $T \rightarrow 0$, although the singular portion will have strong corrections at $T>0$.

\section{B. $Z_{2}$ spin liquid}

We will describe the $Z_{2}$ spin liquid accessed from the $\mathrm{CP}^{N-1}$ model of Section II by condensing a charge- 2 Higgs scalar, $\Phi{ }^{22.47}$ We add a term like $\left|\left(\partial_{\mu}-2 i A_{\mu}\right) \Phi\right|^{2}$ to the full action of the $\mathrm{CP}^{N-1}$ model, and assume we are deep in the phase with $\langle\Phi\rangle \neq 0$. This condensation gaps out the $A_{\mu}$ excitations, and so it is useful to completely integrate out the $A_{\mu}$. The result is an effective action for bosonic spinons $z_{\alpha}$ which describes a bulk transition from a ordered state with non-collinear magnetic order to a $Z_{2}$ spin liquid. This is proposed as a possible model for the triangular lattice antiferromagnet 48.49.50 The effective action obtained by this method has the bulk action

$$
\begin{aligned}
\mathcal{S}_{b}= & \int d^{D} y\left\{\left|\partial_{\mu} z_{\alpha}\right|^{2}+s\left|z_{\alpha}\right|^{2}+\frac{u_{0}}{2}\left(\left|z_{\alpha}\right|^{2}\right)^{2}\right. \\
& \left.+v_{1}\left|z_{\alpha}^{*} \partial_{\mu} z_{\alpha}\right|^{2}+v_{2}\left(z_{\alpha}^{*} \partial_{\mu} z_{\alpha}\right)^{2}+\text { c.c. }\right\},
\end{aligned}
$$

and an impurity term which descends from its $\mathrm{U}(1)$ charge

$$
\begin{aligned}
& \mathcal{S}_{\mathrm{imp}}=\int d\left\{\lambda_{1} z_{\alpha}^{*}(x=0, \tau) \partial_{\tau} z_{\alpha}(x=0, \tau)\right. \\
&\left.+\lambda_{2}\left|z_{\alpha}(x=0, \tau)\right|^{2}\right\} .
\end{aligned}
$$

Here the coefficient $\lambda_{1}$ is proportional to the impurity charge $Q$, but the value of $\lambda_{1}$ is not quantized. An initial derivation does not immediately yield the $\lambda_{2}$ term, but it is clearly allowed by the symmetries and is evidently more relevant than the $\lambda_{1}$ term: it represents a potential scattering term for the bulk spinons. The critical point of the bulk theory alone is a CFT, and it is expected that the $v_{1,2}$ are irrelevant at this critical point, leading to global $\mathrm{O}(2 N)$ symmetry 48,51 . However, as we will see below, the perturbations in $\mathcal{S}_{\text {imp }}$ are not exactly marginal.

It is useful to compare this theory with that of the recent work of Florens et al. ${ }^{18}$ Unlike us, they include an explicit spin degree of freedom at the impurity. In our approach, such a situation would be appropriate when the couplings $\lambda_{1,2}$ are large enough to bind a spinon in the ground state at the impurity. In a $Z_{2}$ spin liquid, there are no long range gauge forces (the $Z_{2}$ gauge forces are short range), unlike the situation in Section IIE and so it is not required that the ground state have non-zero spin for a vacancy impurity, e.g., $\mathrm{Zn}$ on a $\mathrm{Cu}$ site. For a magnetic impurity, such as $\mathrm{Ni}$ on a $\mathrm{Cu}$ site, a finite spin ground state may be more likely, but is also not required when the bulk system is in a gapped $Z_{2}$ spin liquid. We will restrict our discussion here to the small $\lambda_{1,2}$ region of (4.2) +4.3 , and this is equivalent to being in the Kondo-screened phases of Florens et al. ${ }^{18}$.

Let us now describe the phases of $\mathcal{S}_{b}+\mathcal{S}_{\text {imp }}$ as the value of the tuning parameter is scanned.

For $s \gg 0$, we have the gapped spin liquid phase in the bulk. The bulk spinons will experience a potential from the $\lambda_{1,2}$ terms, and in the attractive case in $d=2$, such a potential always has a bound state. However, the binding energy can be less than the bulk spin gap, and hence the ground state remains a singlet and the spingap is preserved. All magnetic response functions will therefore be exponentially small at low $T$.

At the bulk critical point, we need to examine the scaling dimensions of the perturbations in $\mathcal{S}_{\mathrm{imp}}$. In the $\epsilon=4-D$ expansion, simple power-counting shows that 
both $\lambda_{1,2}$ are irrelevant. Consequently, we do not expect a Curie spin susceptibility, but a suppressed response determined by the scaling dimensions of $\lambda_{1,2}$. In the $D=2+\epsilon$ expansion, ${ }^{16}$ we work with the constraint $\left|z_{\alpha}\right|^{2}=1$, and hence the $\lambda_{2}$ term disappears. The $\lambda_{1}$ term is marginal at the tree level, and a more complete renormalization group analysis is needed to understand the flow of $\lambda$; this, however, is beyond the scope of the present paper.

Finally, for $s \ll 0$, in the magnetically ordered phase with collinear order, we can carry out an analysis of the magnetic properties as in Section IID We apply the ansatz in Eq. (2.51) to $\mathcal{S}_{b}+\mathcal{S}_{\text {imp }}$ above, and compute all physical properties as a perturbation series in $u_{0}, v_{1,2}$ and $\lambda_{1,2}$. The result shows that a spatial dependence of the non-collinear order and the uniform (ferromagnetic) magnetization is induced near the impurity by $\lambda_{1,2}$. The uniform magnetization requires the 'particle-hole' symmetry breaking term $\lambda_{1}$, and is non-zero even in the absence of an applied magnetic field. However, unlike the situation in Section IID this ferromagnetic moment is not quantized, and takes a value dependent upon the bare values of the couplings: this is because spin rotation symmetry is completely broken in the non-collinear ordered phase ${ }^{50}$

\section{CONCLUSIONS}

Let us summarize the basic physical characteristics of the impurity response of the various spin liquid states considered in this paper:

(i) Néel-VBS transition: The impurity characteristics across this transition are qualitatively very similar to those found for impurities in confining antiferromagnets,,$\frac{7.9 .16}{.}$ although the underlying field theory is quite different, as are all the exponents and scaling functions. The spin-gap phase has the conventional Curie response of a local moment at the impurity; for the confining antiferromagnets this local moment is induced by confinement physics, while for the Néel-VBS transition it is induced by the logarithmic 'Coulomb' interaction associated with the U(1) gauge field. The Néel-VBS quantum critical point has an anomalous Curie response, with a Curie constant $\mathcal{C}$ specified in Eq. (2.37) to leading order in the $\epsilon=4-D$ expansion. The response of the Néel phase is as found in the earlier case: a spatial varying staggered and uniform moment is found near the impurity, with the moment in the latter quantized as in Eq. (2.57). The Knight shift has both staggered and uniform components, with its behavior in the vicinity of the impurity specified by the impurity exponents determined in Section IIB At the critical point, the induced staggered moment is much stronger than the uniform magnetization component and thus represents the dominating response to an external field.

(ii) Staggered flux spin liquid: This spin liquid is generically critical, and so has an anomalous Curie response; the Curie constant is given, to the leading order in the $1 / N_{f}$ expansion, by Eq. (3.24). The Knight shift now has only a uniform component, but no staggered component in the scaling limit. The absence of any staggered Knight shift is a defining characteristic of the $\mathrm{sF}$ spin liquid, and is a consequence of its large emergent symmetry ${ }^{37}$ There is a large number of competing order parameters, and the leading impurity action $\mathcal{S}_{\text {imp }}$ has no natural way of choosing among them, leading to a response which is restricted to the ferromagnetic moment alone. The behavior of the ferromagnetic Knight shift upon approaching the impurity is specified by the impurity exponent (3.14) found in Section IIC to the leading order in $1 / N_{f}$, the Fourier transform of the ferromagnetic Knight shift is computed in Sect. IIID

(iii) Spinon Fermi surface: This case has a finite magnetic response as $T \rightarrow 0$, unlike the divergent Curie susceptibility of the $\mathrm{sF}$ phase. The response decays with a power law away from the impurity, with the dominant response being at the $2 k_{F}$ wavevector.

(iv) $Z_{2}$ spin liquid: The spin liquid itself has an exponentially suppressed impurity response, because the spin gap is preserved in the presence of the impurity. The proximate ordered non-collinear state has a response similar to that of the Néel state, but with no quantization of the total magnetization.

Apart from their applications to spin liquids, the results in Section [III also have a direct application to the physics of charged impurities in two-dimensional graphene. It is well known that the low energy electronic excitations in graphene are described by 2 species of Dirac fermions. There is no fluctuating gauge field $A_{\mu}$ as in Eq. (3.1). However in the presence of charged impurity, the three-dimensional Coulomb potential the electrons experience has the form $C / r$, where $C$ is a constant. Interestingly, this is exactly the form of the static potential found in Section III in the presence of an impurity, where $\left\langle A_{\tau}\right\rangle \sim 1 / r$. Thus, as long as we ignore quantum-electrodynamic loop corrections, the results of Section III apply also to graphene; specifically, in Fig. [5] (a) and (b) apply to graphene while (c)-(h) do not. One of our important results for this system was Eq. (3.9): that the induced charge density due to the Coulomb potential is a delta function, with $Q$ a non-trivial universal function of $C$. This results therefore applies also to graphene. It disagrees with the earlier result of Ref. 52, which found a $1 / r^{2}$ decay in the induced charge density. We believe their results suffers from a cavalier treatment of the ultraviolet cutoff, which does not preserve gauge invariance.

We now discuss possibilities for future theoretical work.

For the $\mathrm{sF}$ and $\mathrm{CP}^{N-1}$ spin liquids, an alternative $1 / N_{f}$ (or respectively $1 / N$ ) expansion could give a useful complementary picture. We have used an expansion here in which the $N_{f} \rightarrow \infty$ was taken at fixed $Q$. However, it is also possible to study the limit $N_{f} \rightarrow \infty$ limit at fixed $Q / N_{f}$. The saddle-point equations are straightforward 
to obtain for this case, but much more difficult to solve: they have the advantage of including the spinon-impurity 'Coulomb' interaction at all orders already at $N_{f}=\infty$, and so allow for spinon states below, or at the edge of, the bulk spinon continuum. We will report on the results of such an analysis in future work.

It would also be useful to have a more complete understanding of the spinon Fermi surface case, by extending the work of Refs. 45 46 to determine the gauge-invariant response functions near a single impurity.

Finally, we comment on the experimental implications of our work.

For the cuprates, NMR experiments 29.30 show a large Knight shift response at the Néel wavevector in the vicinity of the impurity, and strong temperature-dependent enhancement of such correlations. These features are consistent with the Néel-VBS transition considered in Section III but also with the transition in dimerized antiferromagnets considered earlier ${ }^{7.9 .16}$ The absence of a staggered response for the $\mathrm{sF}$ case is potentially a serious deficiency of the model of Section IIII It remains to be seen if corrections to scaling (such as those in Eq. (3.11)) can remedy the situation.

For the organic Mott insulator $\kappa-(\mathrm{ET})_{2} \mathrm{Cu}_{2}(\mathrm{CN})_{3}$, the NMR Knight shift ${ }^{33}$ has an appreciable $T$ independent component at low $T$. This can potentially be fit either by the spinon Fermi surface, or by a weakly magnetically ordered state.

\section{Acknowledgments}

We thank A. Castro Neto, M. Hermele, Y. B. Kim, T. Senthil, and M. Vojta for useful discussions. This research was supported by the National Science Foundation under grant DMR-0537077. AK was supported by the grant KO2335/1-1 under the Heisenberg Program of Deutsche Forschungsgemeinschaft.
1 A. C. Hewson, The Kondo problem to Heavy Fermions, Cambridge University Press, Cambridge (1993).

2 P. H. Nozieres and A. Blandin, J. Phys. (Paris) 41, 193 (1980).

3 B. A. Jones, C. M. Varma, and J. W. Wilkins, Phys. Rev. Lett. 61, 125 (1988).

${ }^{4}$ L. Fritz and M. Vojta, Phys. Rev. B 70, 214427 (2004); M. Vojta and L. Fritz, Phys. Rev. B 70, 094502 (2004).

${ }^{5}$ C. L. Kane and M. P. A. Fisher, Phys. Rev. Lett. 68, 1220 (1992).

6 S. Eggert and I. Affleck, Phys. Rev. B 46, 10866 (1992); Phys. Rev. Lett. 75, 934 (1995).

7 S. Sachdev, C. Buragohain, and M. Vojta, Science 286, 2479 (1999).

8 A. M. Sengupta, Phys. Rev. B 61, 4041 (2000).

9 M. Vojta, C. Buragohain, and S. Sachdev, Phys. Rev. B 61, 15152 (2000).

10 S. Sachdev, M. Troyer, and M. Vojta, Phys. Rev. Lett. 86, 2617 (2001).

11 S. Sachdev, Physica C 357, 78 (2001).

12 L. Zhu and Q. Si, Phys. Rev. B 66, 024426 (2002).

13 G. Zaránd and E. Demler, Phys. Rev. B 66, 024427 (2002).

14 Q. Si, S. Rabello, K. Ingersent, and J. L. Smith, Nature 413, 804 (2001).

15 M. Troyer, Prog. Theor. Phys. Supplement 145, 326 (2002).

16 S. Sachdev, and M. Vojta, Phys. Rev. B 68, 064419 (2003).

17 K. H. Höglund and A. W. Sandvik, Phys. Rev. B 70, 024406 (2004).

18 S. Florens, L. Fritz, and M. Vojta, Phys. Rev. Lett 96, 036601 (2006).

19 T. Senthil, A. Vishwanath, L. Balents, S. Sachdev, and M. P. A. Fisher, Science 303, 1490 (2004).

20 T. Senthil, L. Balents, S. Sachdev, A. Vishwanath, and M. P. A. Fisher, Phys. Rev. B 70, 144407 (2004).

21 T. Senthil, L. Balents, S. Sachdev, A. Vishwanath, and M. P. A. Fisher, J. Phys. Soc. Japan 74, Suppl. 1 (2005).

22 N. Read and S. Sachdev, Phys. Rev. Lett. 66, 1773 (1991).
23 X.-G. Wen, Phys. Rev. B 44, 2664 (1991).

24 S. A. Krivenko and G. G. Khaliullin, JETP Lett. 62, 723 (1995); G. Khaliullin, R. Kilian, S. Krivenko, and P. Fulde, Phys. Rev. B 56, 11882 (1997).

25 N. Nagaosa and T.-K. Ng, Phys. Rev. B 51, 15588 (1995).

26 N. Nagaosa and P. A. Lee, Phys. Rev. Lett. 79, 3755 (1997).

27 C. Pépin and P. A. Lee, Phys. Rev. Lett. 81, 2779 (1998); Phys. Rev. B 63, 054502 (2001).

28 Z. Wang and P. A. Lee, Phys. Rev. Lett. 89, 217002 (2002).

29 J. Bobroff, H. Alloul, W. A. MacFarlane, P. Mendels, N. Blanchard, G. Collin, and J.-F. Marucco, Phys. Rev. Lett. 86, 4116 (2001).

30 S. Ouazi, J. Bobroff, H. Alloul, M. Le Tacon, N. Blanchard, G. Collin, M. H. Julien, M. Horvatić, and C. Berthier, Phys. Rev. Lett. 96, 127005 (2006).

31 A. Yazdani, B. A. Jones, C. P. Lutz, M. F. Crommie, and D. M. Eigler, Science 275, 1767 (1997).

32 S. H. Pan, E. W. Hudson, K. M. Lang, H. Eisaki, S. Uchida, J. C. Davis, Nature (London) 403, 746 (2000); E W. Hudson, K. M. Lang, V. Madhavan, S. H. Pan, H. Eisaki, S. Uchida, and J. C. Davis, Nature (London) 411, 920 (2001).

33 Y. Shimizu, K. Miyagawa, K. Kanoda, M. Maesato, and G. Saito, Phys. Rev. B 73, 140407 (2006).

34 S. Sachdev in Quantum magnetism, U. Schollwöck, J. Richter, D. J. J. Farnell and R. A. Bishop eds, Lecture Notes in Physics, Springer, Berlin (2004), cond-mat/0401041

35 W. Rantner and X.-G. Wen, Phys. Rev. Lett. 86, 3871 (2001); Phys. Rev. B 66, 144501 (2002).

36 M. Hermele, T. Senthil, M. P. A. Fisher, P. A. Lee, N. Nagaosa, and X.-G. Wen, Phys. Rev. B 70, 214437 (2004).

37 M. Hermele, T. Senthil, and M. P. A. Fisher, Phys. Rev. B 72, 104404 (2005).

${ }^{38}$ F. S. Nogueira and H. Kleinert, Phys. Rev. Lett. 95, 176406 (2005).

39 B. I. Halperin, T. C. Lubensky, and S.-k. Ma, Phys. Rev. 
Lett. 32, 292 (1974)

40 A. V. Chubukov, S. Sachdev, and J. Ye, Phys. Rev. B 49, 11919 (1994).

41 see, e.g., J. O. Andersen and M. Strickland, e-print hep-ph/0404164, and refereces therein.

42 I. D. Lawrie, Nucl. Phys. B 200, 1 (1982).

43 G. Murthy and S. Sachdev, Nucl. Phys. B 344, 557 (1990).

44 C. Itzykson and J.-B. Zuber, Quantum Field Theory, pg 276, McGraw-Hill, New York (1980).

45 B. L. Altshuler, L. B. Ioffe, and A. J. Millis, Phys. Rev. B 50, 14048 (1994); B. L. Altshuler, L. B. Ioffe, A. I. Larkin, and A. J. Millis, Phys. Rev. B 52, 4607 (1995).

46 Y. B. Kim and A. J. Millis, Phys. Rev. B 67, 085102 (2003).
47 S. Sachdev and N. Read, Int. J. Mod. Phys. B 5, 219 (1991).

48 A. V. Chubukov, T. Senthil and S. Sachdev, Phys. Rev. Lett. 72, 2089 (1994); A. V. Chubukov, S. Sachdev, and T. Senthil, Nucl. Phys. B 426, 601 (1994).

49 S. V. Isakov, T. Senthil, and Y. B. Kim, Phys. Rev. B 72, 174417 (2005).

50 S. Sachdev, Rev. Mod. Phys. 75, 913 (2003).

51 P. Azaria, B. Delamotte, and T. Jolicoeur, Phys. Rev. Lett. 64, 3175 (1990).

52 D. P. DiVincenzo and E. J. Mele, Phys. Rev. B 29, 1685 (1984). 